Prepared for the U.S. Department of Energy under Contract DE-AC05-76RL01830

\title{
Viscoelastic Model for Lung Parenchyma for Multi-Scale Modeling of Respiratory System Phase I: Hypo-Elastic Model for CFD Implementation
}

\author{
AD Freed \\ DR Einstein
}

May 2011

\section{Pacific Northwest}

NATIONAL LABORATORY

Proudly Operated by Battelle Since 1965 


\title{
DISCLAIMER
}

This report was prepared as an account of work sponsored by an agency of the United States Government. Neither the United States Government nor any agency thereof, nor Battelle Memorial Institute, nor any of their employees, makes any warranty, express or implied, or assumes any legal liability or responsibility for the accuracy, completeness, or usefulness of any information, apparatus, product, or process disclosed, or represents that its use would not infringe privately owned rights. Reference herein to any specific commercial product, process, or service by trade name, trademark, manufacturer, or otherwise does not necessarily constitute or imply its endorsement, recommendation, or favoring by the United States Government or any agency thereof, or Battelle Memorial Institute. The views and opinions of authors expressed herein do not necessarily state or reflect those of the United States Government or any agency thereof.

\author{
PACIFIC NORTHWEST NATIONAL LABORATORY \\ operated by \\ BATTELLE \\ for the \\ UNITED STATES DEPARTMENT OF ENERGY \\ under Contract DE-AC05-76RL01830
}

Printed in the United States of America
Available to DOE and DOE contractors from the Office of Scientific and Technical Information,
P.O. Box 62, Oak Ridge, TN 37831-0062;
ph: (865) 576-8401
fax: $(865)$ 576-5728
email: reports@adonis.osti.gov

\begin{abstract}
Available to the public from the National Technical Information Service, U.S. Department of Commerce, 5285 Port Royal Rd., Springfield, VA 22161 ph: (800) 553-6847 fax: $(703) 605-6900$ email: orders@ntis.fedworld.gov online ordering: http://www.ntis.gov/ordering.htm
\end{abstract}

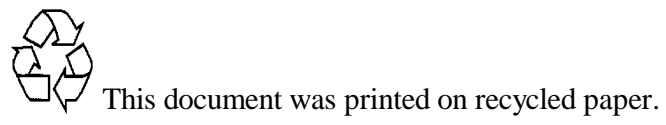




\title{
Viscoelastic Model for Lung Parenchyma for Multi-Scale Modeling of Respiratory System Phase I: Hypo-Elastic Model for CFD Implementation
}

\author{
Alan D. Freed * \\ Clifford H. Spicer Chair in Engineering \\ College of Science, Engineering \& Technology \\ Saginaw Valley State University \\ 202 Pioneer Hall, 7400 Bay Road \\ University Center, MI 48710, USA \\ E-Mail: adfreed@svsu.edu \\ DANIEL R. EINSTEIN \\ Biological Monitoring and Modeling \\ Pacific Northwest National Laboratory \\ 1007 Rogers Street Northwest \\ Olympia, WA 98502, USA \\ E-Mail: daniel.einstein@pnl.gov
}

\section{"Nothing is harder to surmount than a corpus of true but too special knowledge; to reforge the tradition of his forebears is the greatest originality a man can have." \\ C. Truesdell, 1961 [54]}

April 8, 2011

\section{Abstract}

An isotropic constitutive model for the parenchyma of lung has been derived from the theory of hypo-elasticity. The intent is to use it to represent the mechanical response of this soft tissue in sophisticated, computational, fluid-dynamic models of the lung. This demands that the continuum model be accurate, yet simple and efficient. An objective algorithm for its numeric integration is provided. The response of the model is determined for several boundary-value problems whose experiments are used for material characterization. The

* First annual report to Pacific Northwest National Laboratory from Saginaw Valley State University reporting on progress made under grant number 136492. The project described was supported by Award Number R01HL073598 from the National Heart, Lung, and Blood Institute to Pacific Northwest National Laboratory. The content is solely the responsibility of the author and does not necessarily reflect the official views of the National Heart, Lung, and Blood Institute or the National Institutes of Health. 
effective elastic, bulk, and shear moduli, and PoIsson's ratio, as tangent functions, are also derived. The model is characterized against published experimental data for lung. A bridge between this continuum model and a dodecahedral model of alveolar geometry is investigated, with preliminary findings being reported.

\section{Introduction}

This is the first annual report for a two-year project subcontracted to SVSU by PNNL in support of grant R01HL073598 awarded to PNNL by the National Heart, Lung, and Blood Institute within the National Institutes of Health.

The goal for the first year in this contractual effort is to construct a general hypo-elastic theory suitable for lung parenchyma that can be efficiently implemented into computational fluid-dynamics (CFD) codes for the modeling of lung.

The goal of the second year will be to extend this mathematical structure into a hypo-viscoelastic theory for lung.

A long-term objective of this research track is to attempt to construct a linkage between the invariants and parameters of these continuum theories with properties that associate with a microscopic model of the alveolar structure of lung, with some preliminary findings being reported upon herein. Ideally, this would allow low-level models of disease states to be constructed that could then be analytically mapped into a set of continuum parameters, with the resulting continuum model being designed for an efficient implementation within CFD codes.

\subsection{Observations}

The parenchyma of lung consists of the soft tissues that make up the lung. These tissues are the whole of lung except for: the trachea, the bronchi, the bronchioles, and the terminal bronchioles that comprise the airway network, and the arteries and veins of the circulatory system. The soft tissues of the parenchyma are considered to include: the alveoli, the alveolar ducts, the respiratory bronchioles, and the capillary fields that surround them [58], which comprise the bulk of lung tissue. Reviews on the structure and function of lung have been written by Fung $[19,20]$, Weibel \& GiL [57] and West [58].

In a study of alveoli and alveolar ducts, Fung concluded that:

"The regularity of the pulmonary alveolar structure is suggested not only by the numerous histological photographs but also by the patterns of distribution of collagen and elastin fibers in the alveolar walls and mouths. The statistical distributions of the fiber width, curvature, and orientation seem to be spatially uniform. Hence it is reasonable to assume that all alveoli are basically equal and that apparent differences are caused by geometric constraints and by distension, gravity, surface tension, and other loadings."

FUNG [18]

In other words, lung parenchyma, in an averaged sense, is an isotropic material subjected to a heterogeneous deformation/loading field. This conclusion, drawn from histology, supports earlier data obtained by RADFORD [42] from elongation experiments done on strips of dog lung that were extracted from circumferential, radial and vertical orientations. 


\subsection{Objective}

The model being developed is targeted for implementation into OpenFOAM $\cap[21]$, which is a finite-volume CFD code. Consequently, the model needs to be constructed for the Eulerian frame. The independent variables coming from the solver are: the time $t$; the changing volume $\mathrm{d} v$ of each element, i.e., $\partial(\mathrm{d} v(t) / \mathrm{d} V) / \partial t$, wherein $\mathrm{d} V=\mathrm{d} v(0)$; the velocity vector $v$; and the velocity gradient $\boldsymbol{l}=\operatorname{grad} v$, where $\operatorname{grad}=\partial / \partial x$ denotes the gradient of a field in its spatial variable $x$. Given this information, a constitutive model returns the CAUCHY stress $\sigma$, which the solver ensures to be in accordance with physical law through iterative refinements over the assigned field of independent variables.

\subsection{Physics}

The conservation of mass is described by the field equation [25]

$$
\frac{\mathrm{D} \varrho}{\mathrm{D} t}+\operatorname{div}(v) \varrho=0,
$$

where $\varrho$ is the mass density (mass per unit volume), $\mathrm{D} / \mathrm{D} t=\partial / \partial t+\operatorname{grad}() \cdot v$ is the material derivative, and $\operatorname{div} v=\partial \mathrm{v}_{i} / \mathrm{x}_{i}$ is the divergence of the velocity field.

The stress equations of motion (a continuum description of NewTon's second law of motion) are described by the field equation [25]

$$
\varrho \frac{\mathrm{D} v}{\mathrm{D} t}=\not{b}+\operatorname{div} \boldsymbol{\sigma}
$$

where $\mathbb{b}$ is a body force vector, while vector $\operatorname{div} \sigma=\partial \sigma_{i k} / \partial x_{k} e_{i}$ is the divergence of stress. In our study of lung, the gravity vector is a viable body force, viz., $\not{b}=\varrho g$.

The above, two, physical laws can be combined into a single conservation law that reads

$$
\frac{\mathrm{D} \mathbb{P}}{\mathrm{D} t}+\operatorname{div}(v) \mathbb{P}=\not{b}+\operatorname{div} \boldsymbol{\sigma}
$$

wherein the momentum $\mathbb{P}=\varrho v$ per unit volume is the conserved field. The conservation of angular momentum requires a symmetric stress, viz., $\sigma=\sigma^{T}$ [25].

The conservation of linear momentum simplifies to

$$
\dot{p}+\operatorname{div}(v) \mathbb{p}=\not{b}+\operatorname{div} \sigma,
$$

for fluids, because $\operatorname{grad} p=\mathbf{0}$ from $\dot{\varrho}=0$, assuming material incompressibility.

In many situations it is reasonable to neglect the body force caused by gravity, i.e., $\not{b}=\varrho g$ can be taken to be zero.

Neither of these two simplifying assumptions can be imposed when studying lung. Lung is highly compressible, and the effect that gravity has on its physiologic function is significant [58].

\subsection{Constitutive Incorporation}

A novel approach was introduced by GreENSHIELDS \& WELLER [21], the creators of OpenFOAM $($, to unify constitutive expressions so that a single solver can be used to satisfy the linear momentum equation (3) in applications of fluid/solid interaction. What they did, effectively, was to introduce a master function for the divergence of stress, where each material model is expressed as a function of the velocity gradient.

Their original function toggles between the Newtonian fluid (where stress is already a function of $\boldsymbol{l}=\operatorname{grad} v)$ and the Hookean solid (which needs to be differentiated in order to get it expressed in terms of $\boldsymbol{l}$, and then integrated to return the updated state of stress). Material selection depends upon the particular material that resides at a given GAuss point within a volume element belonging to some finite-volume mesh.

In essence, what Greenshields \& Weller 
did was to introduce a function $\operatorname{div}(\sigma)$ so that

$$
\operatorname{div}(\boldsymbol{\sigma})(\boldsymbol{l})= \begin{cases}\operatorname{div}(\boldsymbol{\sigma}(\boldsymbol{d})) & \text { if fluid, } \\ \operatorname{div}\left(\int \dot{\boldsymbol{\sigma}}(\boldsymbol{d}) \mathrm{d} t\right) & \text { if solid }\end{cases}
$$

wherein $\boldsymbol{d}=\operatorname{sym} \boldsymbol{l}$ is the strain rate, $\dot{\sigma}$ is an objective measure of stress rate, and $\int \dot{\sigma} \mathrm{d} t$ is its reciprocal operator, viz., an objective integration for stress. Function $\boldsymbol{\sigma}(\boldsymbol{d})$ represents the constitutive response of a fluid, while function $\dot{\sigma}(\boldsymbol{d})$ denotes the constitutive response of a solid. Collectively, they allow Eq. (3) to be re-expressed as

$$
\frac{\mathrm{D} \mathbb{P}}{\mathrm{D} t}+\operatorname{div}(v) \mathbb{P}=\not{b}+\operatorname{div}(\boldsymbol{\sigma})(\boldsymbol{d}),
$$

where now everything is nicely written in terms of the velocity vector, not the displacement vector, which offers many advantages that the developers of OpenFOAM $®$ utilized in its construction.

All good ideas are simple when understood, but the path traversed to enlightenment is often tortuous. Such was the case here.

\subsection{Constitutive Approach}

Numerous investigators have proposed a variety of hyper-elastic material models for describing the passive response of lung parenchyma, e.g., Frankus \& Lee [9, 31], Fung [17], KarakaPlan, Bieniek \& Skalak [27], and Vawter [56]. These are models where stress is described as a function of strain, i.e., $\sigma(\boldsymbol{\epsilon})$, but the CFD formulation of GREENSHIELDS \& WELLER [21] wants solid models whose functional form looks like $\dot{\sigma}(\boldsymbol{d})$, which are hypo-elastic in structure. Here the literature is wanting.

In the author's textbook [13], a hypo-elastic theory that is isotropic in stress and strain rate is put forward for incompressible soft solids. Lung, however, is compressible. The author's theory must therefore be extended to address material compressibility. The outcome is a constitutive model that TRUESDELL [52] called a hypo-elastic material of grade one.

In what follows, a hypoelastic constitutive equation is derived that is isotropic in stress and strain rate, and whose functional form looks like

$$
\stackrel{\circ}{\sigma}=\oplus(\sigma,|\mathbf{F}|): d,
$$

with $\stackrel{\circ}{\sigma}$ being TruesDell's [51] stress rate, cf. Eq. (18), and $|\mathbf{F}|=\int \partial\left(\mathrm{d} v\left(t^{\prime}\right) / \mathrm{d} V\right) / \partial t^{\prime} \mathrm{d} t^{\prime}$ is the Jacobian of deformation. Consequently, the idea expressed in Eqs. $(5 \& 6)$ continues to apply provided that $\operatorname{div}(\sigma)(\sigma,|\mathbf{F}|, \boldsymbol{d})$.

The forth-order tensor $\oplus$ is the hypo-elastic tangent modulus, whose construction is the main objective of this first report. It is a function in stress and the changing volume. The actual tangent modulus $\theta$ can be found in Eq. (29). An objective algorithm for the numeric integration of such a hypo-elastic solid is provided in $\$ 2.2 .2$.

This tangent modulus is derived from a potential function constructed in the Lagrangian frame, where the physics are more intuitive. The ensuing model is then pushed forward into the Eulerian frame so that it can be used in OpenFOAM $\cap$. Before the details of this formalism can be described, however, some tensorial fields need to be defined. Details that may be missing here can be found in the author's textbook [13].

\section{Physical Fields}

Lagrangian fields are described in terms of a position location $\mathbb{X}$ that points to some continuum particle $P$ in its material configuration $\Omega_{0}$ associated with a reference time $t_{0}$ that, because time is relative, is translated to $t_{0}=$ 0 . Eulerian fields are described in terms of a position variable $x=\mathcal{X}(\mathbb{X}, t)$ that locates this 
same particle $\mathbb{P}$ in the body's spatial configuration $\Omega$, which associates with current time $t$. The path traversed by this particle can be described in terms of a mapping function: the particle's motion $\mathcal{X}$ through ambient space $\mathbb{R}^{3}$, whose existence is postulated in the law of continuous media - the fundamental postulate of continuum mechanics.

Lagrangian fields defined over $\Omega_{0}$ can be mapped (pushed forward) into Eulerian fields defined over $\Omega$. This mapping is reversible in that Eulerian fields can be also mapped (pulled back) into Lagrangian fields. Each tensor index can be mapped in one of two ways: covariant (i.e., as a normal to a surface) or contravariant (i.e., as a tangent to a curve). A characteristic property of every physical field is the transfer law that it obeys when being mapped between configurations.

\subsection{Strains}

There are two physical descriptions for strain that are defined. One is covariant; the other is contravariant. One is well known; the other is not. They are duel measures of strain. One measures the change in distance between two material points; the other measures the change in distance between two, non-intersecting, material surfaces.

In the Lagrangian frame, these are: the covariant strain measure of GREEN

$$
\boldsymbol{E}=\frac{1}{2}(\boldsymbol{C}-\mathbf{I})=\frac{1}{2}\left(\mathbf{F}^{T} \mathbf{F}-\mathbf{I}\right),
$$

and the contravariant strain measure of LoDGE

$$
\boldsymbol{E}=\frac{1}{2}\left(\mathbf{I}-\boldsymbol{C}^{-1}\right)=\frac{1}{2}\left(\mathbf{I}-\mathbf{F}^{-1} \mathbf{F}^{-T}\right),
$$

where $\mathbf{F}=\partial \not \chi \chi X, t) / \mathbb{X}$ is the deformation gradient, and $\boldsymbol{C}$ and $\boldsymbol{C}^{-1}$ are the deformation metrics of GreEn and CaUCHY, respectively.

If $\mathrm{d} S$ and $\mathrm{d} s$ are the initial and current distances separating two, neighboring, material points $P$ and $P^{\prime}$ that reside in both $\Omega_{0}$ and $\Omega$, and if $\mathrm{d} H$ and $\mathrm{d} h$ are the initial and current distances separating two, neighboring, material surfaces $\delta$ and $\delta^{\prime}$ to which $P$ and $P^{\prime}$ belong, as shown in Fig. 1, then in any given direction $\mathbb{N}$ of unit length, i.e., $\|\mathbb{N}\|=1$, one finds that

$$
\begin{gathered}
\frac{(\mathrm{d} s)^{2}-(\mathrm{d} S)^{2}}{2(\mathrm{~d} S)^{2}}=\mathbb{N} \cdot \boldsymbol{E} \mathbb{N} \\
\frac{(\mathrm{d} h)^{2}-(\mathrm{d} H)^{2}}{2(\mathrm{~d} h)^{2}}=\mathbb{N} \cdot \boldsymbol{E} \mathbb{N} .
\end{gathered}
$$

These strain measures are defined in accordance with the geometry of RIEMANN.

These strain fields push forward into the Eulerian frame $\Omega$ which, in turn, pull back into the Lagrangian frame $\Omega_{0}$ according to the maps

$$
\begin{aligned}
\mathbf{e} & =\mathbf{F}^{-T} \boldsymbol{E} \mathbf{F}^{-1}, & \boldsymbol{E} & =\mathbf{F}^{T} \mathbf{e} \mathbf{F}, \\
\boldsymbol{e} & =\mathbf{F} \boldsymbol{E} \mathbf{F}^{T}, & \boldsymbol{E} & =\mathbf{F}^{-1} \boldsymbol{e} \mathbf{F}^{-T},
\end{aligned}
$$

where $\mathbf{e}$ and $\boldsymbol{e}$ are the Almansi and Signorini strains, respectively. These strains obey different maps because $\boldsymbol{E}$ and $\mathbf{e}$ are covariant fields, while $\boldsymbol{E}$ and $\boldsymbol{e}$ are contravariant fields. Consequently, strains $\boldsymbol{E}$ and e represent the same physical property, they are just defined over different configurations. Likewise, strains $\mathcal{E}$ and $\boldsymbol{e}$ represent the same physical measure of strain-a property that happens to be distinct from the strain measured by $\boldsymbol{E}$ and $\mathbf{e}$. Each of these strain pairings is bijective.

As a matter of notation, Lagrangian fields are written in upper case, while Eulerian fields are written in lower case, for the most part.

Strain rate is a different beast altogether. It does not obey a bijective map. This has relevance when constructing rate-based theories, like hypo-elasticity, and is a contributing factor for why this author constructs his theories in the Lagrangian frame.

When one takes the time derivative of a Lagrangian field (i.e., at a fixed particle in the body), and then maps it into the Eulerian frame, one gets the objective rates of OLDRoYd [38], 

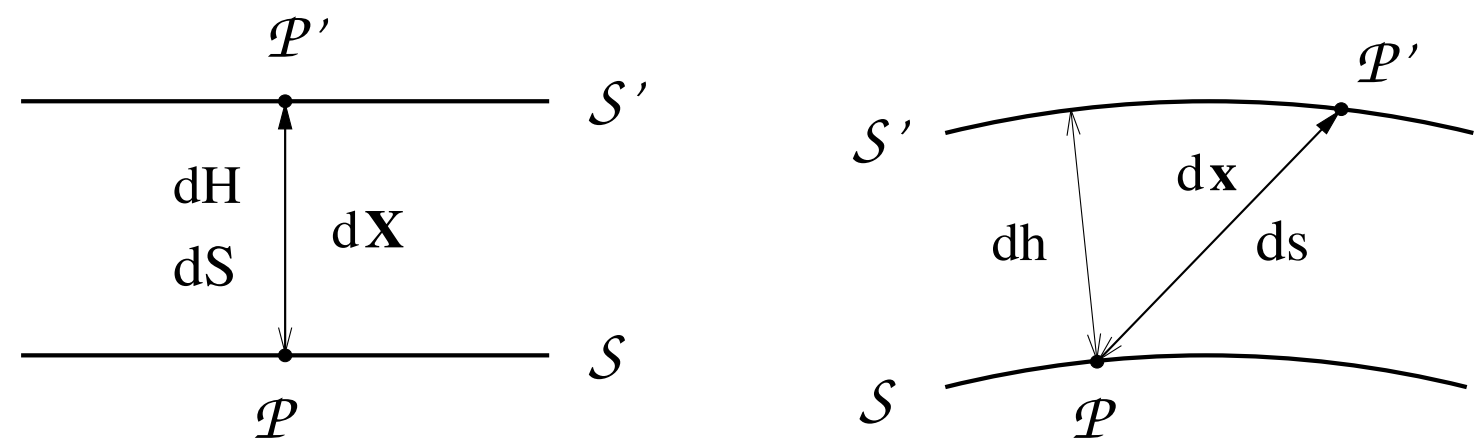

$t_{0}$

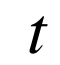

Figure 1: Non-intersecting material surfaces $\delta$ and $\delta^{\prime}$ containing particles $P$ and $\boldsymbol{P}^{\prime}$ deform from an initial configuration at time $t_{0}$ into a final configuration at time $t$. The initial separation of the surfaces $\mathrm{d} H$ deforms to $\mathrm{d} h$ in a manner that is distinct form the separation of the particles going from $\mathrm{d} S$ to $\mathrm{d} s$ as $\mathrm{d} \mathbb{X}$ goes to $\mathrm{d} x$.

a.k.a. as their LIE derivatives [39]. For the strain fields given in Eq. (11), these rates are

$$
\begin{aligned}
& \stackrel{\nabla}{\mathbf{e}}=\mathbf{F}^{-T} \dot{E} \mathbf{F}^{-1}, \quad \dot{E}=\mathbf{F}^{T} \stackrel{\nabla}{\mathbf{e}} \mathbf{F}, \\
& \stackrel{\Delta}{\boldsymbol{e}}=\mathbf{F} \dot{\boldsymbol{E}} \mathbf{F}^{T}, \quad \dot{\boldsymbol{E}}=\mathbf{F}^{-1} \stackrel{\Delta}{\boldsymbol{e}} \mathbf{F}^{-T},
\end{aligned}
$$

where $\nabla$ and $\Delta$ denote OLDRoyd's lower- and upper-convected derivatives, respectively. The former applies to covariant tensor fields, while the latter applies to contravariant tensor fields. These two derivatives are calculated differently.

Strains are special fields in that their LIE derivatives are equivalent, viz.,

$$
\stackrel{\nabla}{\mathrm{e}}=\boldsymbol{d} \quad \& \quad \stackrel{\Delta}{\boldsymbol{e}}=\boldsymbol{d},
$$

and therefore, in the Eulerian frame $\Omega$,

$$
\stackrel{\nabla}{\mathbf{e}} \equiv \stackrel{\Delta}{\boldsymbol{e}}
$$

Even though $\dot{\boldsymbol{E}} \neq \dot{\boldsymbol{E}}$ in the Lagrangian frame $\Omega_{0}$, these strain rates obey the identity

$$
\dot{\mathcal{E}}=\boldsymbol{C}^{-1} \dot{\boldsymbol{E}} \boldsymbol{C}^{-1},
$$

so they are not unique, either. It is apparent from Eqs. (12-15) that strain-rate mappings are many-to-one, and therefore, they are not bijective, viz., they are not one-to-one and onto. Derivations of these formulæ can be found in the author's textbook [13].

\subsection{Stresses}

Strains are absolute tensor fields in that their field-transfer maps do not depend upon the Jacobian of the mapping, viz., the determinant $|\mathbf{F}|$. Stress, on the other hand, does depend on this Jacobian; specifically,

$$
\begin{aligned}
& \boldsymbol{S}=|\mathbf{F}| \mathbf{F}^{-1} \boldsymbol{\sigma} \mathbf{F}^{-T}, \\
& \boldsymbol{\sigma}=|\mathbf{F}|^{-1} \mathbf{F} \boldsymbol{S} \mathbf{F}^{T},
\end{aligned}
$$

where $\sigma$ is the Cauchy stress, and $S$ is the second Piola-Kirchioff stress. These maps associate with a contravariant tensor field of weight one [34]. The determinant arises naturally here, because the normal to an infinitesimal area maps as a relative covariant vector of weight minus-one; namely, $\pi \mathrm{d} a=|\mathbf{F}| \mathbf{F}^{-T} \mathbb{N} \mathrm{d} A$, where $\mathrm{d} A$ is the area of an infinitesimal material surface with normal $\mathbb{N}$ assigned in the reference configuration $\Omega_{0}$ that then gets pushed forward into the current configuration $\Omega$ as the area $\mathrm{d} a$ to a surface with unit normal $\pi$. This is known as NANSON's theorem.

Taking the derivative of $S$ with respect to time $t$ at a fixed particle $\mathbb{X}$ in configuration $\Omega_{0}$, using the upper expression of Eq. (16), which is the physical description for stress in the Lagrangian frame, while preserving the integrity 
of the field-transfer map for stress, leads to

$$
\begin{aligned}
& \dot{\boldsymbol{S}}=|\mathbf{F}| \mathbf{F}^{-1} \stackrel{\circ}{\sigma} \mathbf{F}^{-T}, \\
& \stackrel{\circ}{\sigma}=|\mathbf{F}|^{-1} \mathbf{F} \dot{S} \mathbf{F}^{T},
\end{aligned}
$$

wherein

$$
\stackrel{\circ}{\sigma}=\mathrm{D} \boldsymbol{\sigma} / \mathrm{D} t+\operatorname{tr}(\boldsymbol{l}) \boldsymbol{\sigma}-\boldsymbol{l} \boldsymbol{\sigma}-\boldsymbol{\sigma} \boldsymbol{l}^{T}
$$

is commonly referred to as TRUESDELL's [51, 53] measure for stress rate. ${ }^{*}$ To arrive at this stress rate, the following identities have been used:

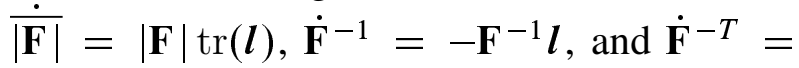
$-\boldsymbol{l}^{T} \mathbf{F}^{-T}$, along with the chain rule.

TruesDell's stress rate, and the time rate of change of the second Piola-KirchHoff stress, are the same physical measure for stress rate, they are just defined over different configurations. (Recall that CUACHY stress and the second Piola-KirchHoff stress are the same physical measures for stress, so this pairing of stress and stress-rate fields is physically consistent.) In practice, one does not compute the stress rate via Eq. (18); rather, its value is specified through a constitutive equation, which must then be integrated.

\subsubsection{Objective Integration of Stress Rate}

To acquire an admissible integration for stress in the Eulerian frame $\Omega$, it is easiest to integrate stress rate in the Lagrangian frame $\Omega_{0}$ at a fixed particle $\mathbb{X}$ over some interval $[0, t]$ of interest; in other words, it is simplest to first integrate

$$
\boldsymbol{S}(\mathbb{X}, t)=\boldsymbol{S}(\mathbb{X}, 0)+\int_{0}^{t} \dot{\boldsymbol{S}}\left(\mathbb{X}, t^{\prime}\right) \mathrm{d} t^{\prime}
$$

whose solution one then pushes forward into the Eulerian frame for use. Here $S(\mathbb{X}, 0)$ designates a residual state of stress, if it exists. One integrates in the reference configuration $\Omega_{0}$, because the Lagrangian position vector $\mathbb{X}$ associates with the same physical particle $P$ over the entire interval of integration $[0, t]$
$[12,13]$. A different particle resides at the Eulerian location $x$ at each instant over a time interval; therefore, an inadmissible integration within $\Omega$ could easily be constructed-one that retains no physical meaning.

The pushing-forward process begins by translating the material variables in the integrand into their spatial counterparts by substituting the field-transfer map from the first line in Eq. (17) into the integrand. This leads to an intermediate result of

$$
\begin{aligned}
& S(t)=S(0) \\
& \quad+\int_{0}^{t}\left|\mathbf{F}\left(t^{\prime}\right)\right| \mathbf{F}^{-1}\left(t^{\prime}\right) \stackrel{\circ}{\sigma}\left(t^{\prime}\right) \mathbf{F}^{-T}\left(t^{\prime}\right) \mathrm{d} t^{\prime},
\end{aligned}
$$

where the integrand is still located in the reference frame, viz., it is still $\dot{\boldsymbol{S}}$, it is just expressed in terms of spatial fields instead of material fields. Now, one pushes the whole expression forward into the Eulerian frame in accordance with the field-transfer map stated in the second line of Eq. (16), which gives

$$
\begin{aligned}
\sigma(t) & =|\mathbf{F}|^{-1} \mathbf{F} \boldsymbol{S}(0) \mathbf{F}^{T} \\
+ & |\mathbf{F}|^{-1} \mathbf{F} \int_{0}^{t}|\mathbf{F}| \mathbf{F}^{-1} \stackrel{\circ}{\sigma} \mathbf{F}^{-T} \mathrm{~d} t^{\prime} \mathbf{F}^{T}
\end{aligned}
$$

All fields inside the integrand are evaluated at time $t^{\prime}$, and all fields outside the integrand are evaluated at time $t$, except for a residual stress $S(0) \equiv \sigma(0)$ that belongs to the initial state. Equation (21) is an objective integration of stress rate in the Eulerian frame. Oldroyd [38] refers to this as a convected integration-the inverse of his convected differentiation. We *. Actually, this objective rate appeared earlier in OLDRoYd's [38] paper, in a general setting. OldRoyd's interest was in viscoelastic liquids that are incompressible, in which case $\operatorname{tr} \boldsymbol{l}=0$, and as such, Eq. (18) takes on the form of what is now commonly referred to as OLDROYD's upper-convected derivative of stress, viz., $\stackrel{\Delta}{\sigma}=\mathrm{D} \boldsymbol{\sigma} / \mathrm{D} t-\boldsymbol{l} \boldsymbol{\sigma}-\boldsymbol{\sigma} \boldsymbol{l}^{T}$. 
reaffirm that TRUESDELL's stress rate is a convected derivative, in the sense of OLDROYD; it is for a relative contravariant tensor whose weight of mapping is one.

Equation (21) is an analytic result. Our application requires a numeric approximation to this integral based on an iterative scheme that would be suitable for advancing the solution in

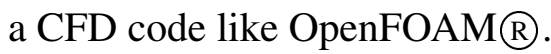

\subsubsection{Numerical Integration for Stress}

In this algorithm, what one is doing is using configuration $\Omega_{n-1}$ as the reference frame, and configuration $\Omega_{n}$ as the current frame. This is commonly referred to in the literature as an updated Lagrangian formulation [2].

Equation (21) can be readily generalized into a form that is easily implemented into an iterative numeric scheme; specifically,

$$
\begin{aligned}
& \sigma_{n}=|\widehat{\mathbf{F}}|^{-1} \widehat{\mathbf{F}} \sigma_{n-1} \hat{\mathbf{F}}^{T} \\
& +|\widehat{\mathbf{F}}|^{-1} \hat{\mathbf{F}} \int_{t_{n-1}}^{t_{n}}|\widetilde{\mathbf{F}}| \widetilde{\mathbf{F}}^{-1} \stackrel{\circ}{\sigma}\left(t^{\prime}\right) \widetilde{\mathbf{F}}^{-T} \mathrm{~d} t^{\prime} \widehat{\mathbf{F}}^{T},
\end{aligned}
$$

with an initial condition of $\sigma_{0}=\sigma(0) \equiv S(0)$ which, if it exists, represents a residual stress. Here use has been made of the generalized deformation gradients

$$
\begin{aligned}
& \widehat{\mathbf{F}}=\mathbf{F}\left(t_{n-1}, t_{n}\right)=\mathbf{F}\left(t_{n}\right) \mathbf{F}^{-1}\left(t_{n-1}\right), \\
& \widetilde{\mathbf{F}}=\mathbf{F}\left(t_{n-1}, t^{\prime}\right)=\mathbf{F}\left(t^{\prime}\right) \mathbf{F}^{-1}\left(t_{n-1}\right),
\end{aligned}
$$

wherein the former describes the increment of deformation between $\Omega_{n-1}$ and $\Omega_{n}$ (in terms of a pair of the global deformations: $\Omega_{0} \rightarrow \Omega_{n-1}$ and $\Omega_{0} \rightarrow \Omega_{n}$ ), while the latter describes another deformation increment between $\Omega_{n-1}$ and $\Omega$, wherein $t_{n-1} \leq t^{\prime} \leq t_{n}$.

To illustrate how this works, consider the following method, which is suitable for implementation into a CFD code. Here the trapezoidal rule is used, with the rectangular (forward EULER) rule being called upon to start the process on the first pass of the solver at any given integration step. Such an algorithm would look like:

Algorithm 1

$$
\begin{aligned}
& h=t_{n}-t_{n-1} \text {; } \\
& \text { IF firstiteration THEN } \\
& \Delta \sigma=h \oplus\left(\sigma_{n-1},|\mathbf{F}|_{n-1}\right): d_{n-1} ; \\
& \sigma_{n}^{p}=|\widehat{\mathbf{F}}|^{-1} \hat{\mathbf{F}}\left(\sigma_{n-1}+\Delta \boldsymbol{\sigma}\right) \hat{\mathbf{F}}^{T} ; \\
& \text { ELSE } \\
& \sigma_{n}^{p}=\sigma_{n} ; \quad(* \text { from previous iterate } *) \\
& \text { ENDIF; } \\
& \Delta \sigma_{\ell}=\frac{1}{2} h \oplus\left(\sigma_{n-1},|\mathbf{F}|_{n-1}\right): d_{n-1} ; \\
& \Delta \sigma_{u}=\frac{1}{2} h \oplus\left(\sigma_{n}^{p},|\mathbf{F}|_{n}\right): d_{n} ; \\
& \sigma_{n}=|\widehat{\mathbf{F}}|^{-1} \hat{\mathbf{F}}\left(\sigma_{n-1}+\Delta \sigma_{\ell}\right) \hat{\mathbf{F}}^{T}+\Delta \sigma_{u} ; \\
& \text { RETURN } \sigma_{n} \text {. }
\end{aligned}
$$

This is but one of many numerical schemes that one could devise to solve Eq. (22). The endcorrection methods of GrEGory, for example, could be called upon for more accurate results, assuming that $\stackrel{\circ}{\sigma}$ is sufficiently smooth [3].

In the above algorithm, $\stackrel{\circ}{\sigma}$ has been replaced by $\Theta: \boldsymbol{d}$, in accordance with the constitutive hypothesis presented in Eq. (7).

Integrand evaluations made at the lower limit of integration $t_{n-1}$ associate with $\tilde{\mathbf{F}}=$ I. Similarly, integrand evaluations made at the upper limit of integration $t_{n}$ associate with $\widetilde{\mathbf{F}}=\widehat{\mathbf{F}}$. This is why stress quantities associated with state $t_{n-1}$ get pushed forward into state $t_{n}$, in accordance with the field-transfer map for stress; whereas, there is no need to push those stress quantities that are already affiliated with state $t_{n}$.

Failure to push forward any contribution that accumulates anywhere other than at the upper limit of integration with an appropriate mapping, e.g., the push forward of the initial 
stress $\sigma_{n-1}$ in Eq. (22), will lead to a nonobjective integrator. The appropriate generalized deformation gradient to be used at any given quadrature node of integration will depend upon where in the interval $\left[t_{n-1}, t_{n}\right]$ the integrand is being sampled, which is determined by the quadrature rule of the selected integrator. In the case of multi-step methods, the quadrature rule would also sample states prior to $t_{n-1}$, e.g., at $t_{n-2}$, whose contributions would need to be pushed forward in an appropriate manner.

The previous algorithm for integrating stress assumes that all needed kinematic variables, viz., $\boldsymbol{d}_{n-1}, \boldsymbol{d}_{n},|\mathbf{F}|_{n-1},|\mathbf{F}|_{n},|\hat{\mathbf{F}}|$, and $\hat{\mathbf{F}}$, are known to the solver, and as such, are available to the integrator. This need not be the case.

In OpenFOAM $®$, for example, $\boldsymbol{l}_{n-1}, \boldsymbol{l}_{n}$, $\left.\overline{\mathrm{d} v / \mathrm{d} V}\right|_{n-1}$ and $\left.\overline{\mathrm{d} v / \mathrm{d} V}\right|_{n}$ are supplied by the solver. From these basic kinematic variables one can approximate, via the following algorithm, all kinematic variables required by the preceding algorithm for integrating stress, viz.,

Algorithm 2

$$
\begin{aligned}
|\mathbf{F}|_{n}= & |\mathbf{F}|_{n-1} \\
& +h \frac{1}{2}\left(\left.\overline{\mathrm{d} v / \mathrm{d} V}\right|_{n-1}+\left.\overline{\mathrm{d} v / \mathrm{d} V}\right|_{n}\right) \\
|\hat{\mathbf{F}}|= & |\mathbf{F}|_{n} /|\mathbf{F}|_{n-1} \\
\boldsymbol{d}_{n-1} & =\frac{1}{2}\left(\boldsymbol{l}_{n-1}+\boldsymbol{l}_{n-1}^{T}\right) \\
\boldsymbol{d}_{n}= & \frac{1}{2}\left(\boldsymbol{l}_{n}+\boldsymbol{l}_{n}^{T}\right) \\
\hat{\mathbf{F}}=\mathbf{I} & +h \frac{1}{2}\left(\boldsymbol{l}_{n-1}+\boldsymbol{l}_{n}\right) \\
& +O\left(h^{2} \frac{1}{8}\left(\boldsymbol{l}_{n-1}+\boldsymbol{l}_{n}\right)^{2}\right) .
\end{aligned}
$$

Because $|\mathbf{F}|=\mathrm{d} v / \mathrm{d} V$ with an initial condition of $|\mathbf{F}|_{0}=\mathrm{d} V / \mathrm{d} V=1$, its value can be determined by integrating $\dot{|\mathbf{F}|}=\dot{\mathrm{d} v / \mathrm{d} V}$ using the trapezoidal rule, which is the method selected for use to integrate stress.

The second formula comes from properties of the determinant applied to Eq. (23).
The third and forth formulæ establish $\boldsymbol{d}$ at the beginning and end of the interval, and follow directly from its definition.

The final formula comes from the definition for the velocity gradient, viz., $l=\dot{\mathbf{F}} \mathbf{F}^{-1}$. Rewriting this as $\boldsymbol{l}=\mathrm{d} \ln \mathbf{F} / \mathrm{d} t$, it can be integrated to $\ln \mathbf{F}=\int \boldsymbol{l} \mathrm{d} t$, and then rearranged so that $\mathbf{F}=\exp \left(\int \boldsymbol{l} \mathrm{d} t\right)$. The exponential of a matrix $\mathbf{M}$ has an infinite series expansion of $\exp \mathbf{M}=\mathbf{I}+\mathbf{M}+\frac{1}{2 !} \mathbf{M}^{2}+\frac{1}{3 !} \mathbf{M}^{3}+\cdots$. Because $\hat{\mathbf{F}}$ is defined to be the deformation gradient over the interval $\left[t_{n-1}, t_{n}\right]$, it follows that $\widehat{\mathbf{F}}=\exp \left(\int_{t_{n-1}}^{t_{n}} \boldsymbol{l} \mathrm{d} t\right)$. Again, adopting the trapezoidal rule for integration, it follows that $\mathbf{M}=h \frac{1}{2}\left(\boldsymbol{l}_{n-1}+\boldsymbol{l}_{n}\right)$. Substituting this into the series expansion for $\exp \mathbf{M}$ gives the stated result when truncating after the second term.

As a rule of thumb, the number of terms kept in this series ought to match the order of the integrator being used, which for the trapezoidal rule is two. It is worth mentioning that an application of the CAYLEY-HamiLton theorem fore goes the need to solve matrix products greater than $\mathbf{M}^{2}{ }^{\dagger}$

\section{Hypo-Elasticity}

All of the author's $[10,11,12,13,14]$ investigations into hypo-elasticity as a theory for de-

$\uparrow$. For the interested reader, the first six terms in a series expansion for the exponential of a $3 \times 3$ matrix M sum to:

$$
\begin{aligned}
\exp \mathbf{M}= & \left(1+\frac{1}{3 !} I I I+\frac{1}{4 !} I I I I\right. \\
& \left.+\frac{1}{5 !} I I I\left(I^{2}-I I\right)\right) \mathbf{I} \\
+ & \left(1-\frac{1}{3 !} I I-\frac{1}{4 !}(I I I-I I I)\right. \\
& -\frac{1}{5 !}\left(I I\left(I^{2}-I I\right)-I I I I\right) \mathbf{M} \\
+ & \left(\frac{1}{2 !}+\frac{1}{3 !} I+\frac{1}{4 !}\left(I^{2}-I I\right)\right. \\
& \left.+\frac{1}{5 !}\left(I^{3}-2 I I I+I I I\right)\right) \mathbf{M}^{2}
\end{aligned}
$$

where I, II, and III are the three invariants of M. The 5! terms can be removed for a $5^{\text {th }}$ order approximation, the 4 ! and 5! terms for a $4^{\text {th }}$ order approximation, etc. 
Y.-C. Fung's 1967 Experimental Data

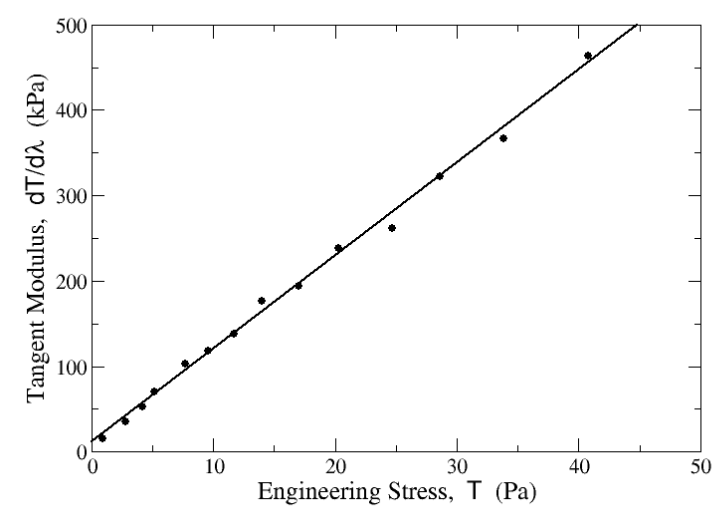

Figure 2: A reconstruction of Fig. 8 from Fung's paper [16], which is an experimental plot for rabbit mesentery, fit here to Eq. (24) with $E=11.7 \mathrm{kPa}$ and $\beta=10.9$.

scribing the mechanical response of soft tissues have been predicated upon Fung's law [16]. Fung proposed the phenomenological model

$$
\frac{\mathrm{dT}(\lambda)}{\mathrm{d} \lambda}=E+\beta \mathrm{T}(\lambda)
$$

wherein $\mathrm{T}$ is the uniaxial engineering stress, $\lambda$ is the stretch, $E$ is Young's modulus, and $\beta$ is referred to as Fung's parameter. He proposed this model based upon the experimental data presented in Fig. 2. Most biomechanicians have sought to extend its hyper-elastic representation, i.e., $\mathrm{T}(\epsilon)=E \beta^{-1}(\exp (\beta \epsilon)-1)$, into 3 -space, with $\epsilon=\lambda-1$ denoting engineering strain. In contrast, this author has extrapolated its hypo-elastic representation, viz., $\dot{\mathrm{T}}(t)=$ $(E+\beta \mathrm{T}(t)) \dot{\lambda}(t)$, into 3-space, which leads to vastly simpler constitutive expressions.

In this report, the tangent modulus for a compressible, isotropic, hypo-elastic solid is derived, and its properties are explored.

\subsection{Constitutive Assumption}

In his textbook [13] the author hypothesized the existence of a potential function $\Phi$ whereby

$$
\dot{\boldsymbol{S}}=\frac{\partial^{2} \Phi(\boldsymbol{S}, \dot{\boldsymbol{E}} ; \boldsymbol{C})}{\partial \dot{\boldsymbol{E}} \partial \dot{\boldsymbol{E}}}: \dot{\boldsymbol{E}}
$$

Each term in potential $\Phi$ must be at most linear in stress to be in accordance with Fung's law (24), and each term must be exactly quadratic in strain rate to be in accordance with TRUESDELL's definition of a hypo-elastic solid [37, 52].

From conjecture (25), one can introduce

$$
\Theta=\frac{\partial^{2} \Phi(\boldsymbol{S}, \dot{\boldsymbol{E}} ; \boldsymbol{C})}{\partial \dot{\boldsymbol{E}} \partial \dot{\boldsymbol{E}}},
$$

where $\Theta=\Theta_{I J K L} \mathfrak{e}_{I} \otimes \mathfrak{e}_{J} \otimes \mathfrak{e}_{K} \otimes \mathfrak{e}_{L}$ is the Lagrangian tangent modulus. It is because GreEN strain $\boldsymbol{E}$ is covariant, while Lodge strain $\mathcal{E}$ is contravariant, that the left-two indices map contravariantly, while the right-two indices map covariantly, viz.,

$$
\begin{aligned}
\theta_{i j k \ell} & =|\mathbf{F}|^{-1} \mathrm{~F}_{i I} \mathrm{~F}_{j J} \Theta_{I J K L} \mathrm{~F}_{K k}^{-1} \mathrm{~F}_{L \ell}^{-1}, \\
\Theta_{I J K L} & =|\mathbf{F}| \mathrm{F}_{I i}^{-1} \mathrm{~F}_{J j}^{-1} \theta_{i j k \ell} \mathrm{F}_{k K} \mathrm{~F}_{\ell L},
\end{aligned}
$$

where $\mathbb{\theta}=\theta_{i j k \ell} \mathfrak{e}_{i} \otimes \mathfrak{e}_{j} \otimes \mathfrak{e}_{k} \otimes \mathfrak{e}_{\ell}$ is the Eulerian tangent modulus given in Eq. (7). The determinant enters into these mappings because Eqs. (25-27) must be consistent with the mappings in Eqs. (12 \& 17) that govern the rates of strain and stress, respectively. Strain and strain-rate are absolute tensors, while stress and stress-rate are relative tensors of weight one; therefore, the tangent modulus must also be a relative field of weight one.

The hypo-elastic tangent moduli in Eqs. (26 $\&$ 27) differ from the hyper-elastic tangent moduli commonly employed in finite elements. Hyper-elastic tangent moduli are absolute tensor fields whose indices map contravariantly [44], while our hypo-elastic tangent moduli are relative tensors with mixed index pairs. 


\subsection{Invariants}

Any material that is an isotropic function of two tensors can be represented in terms of ten scalar invariants [47]. In the case of the $\Phi$ potential in Eq. (26), stress $\boldsymbol{S}$ and strain rate $\dot{\boldsymbol{E}}$ are these two tensor fields. Tensor $\boldsymbol{C}$ is the metric of deformation. It is used for contraction in much the same way that the metric tensor is used in general tensor analysis; specifically, the covariant metric $\boldsymbol{C}$ is used to pull-down contravariant indices, while its inverse, the contravariant metric $C^{-1}$, is used to push-up covariant indices. That is why $\boldsymbol{C}$ is separated from $\boldsymbol{S}$ and $\dot{\boldsymbol{E}}$ in Eqs. ( $25 \& 26)$ by a semicolon.

These ten allowable invariants are: the three invariants of stress: $\operatorname{tr}(\boldsymbol{S C}), \operatorname{tr}(\boldsymbol{S C S C})$, and $\operatorname{tr}(\boldsymbol{S C S C S C})$; the three invariants of strain rate: $\operatorname{tr}\left(\boldsymbol{C}^{-1} \dot{\boldsymbol{E}}\right), \operatorname{tr}\left(\boldsymbol{C}^{-1} \dot{\boldsymbol{E}} \boldsymbol{C}^{-1} \dot{\boldsymbol{E}}\right)$, and $\operatorname{tr}\left(\boldsymbol{C}^{-1} \dot{\boldsymbol{E}} \boldsymbol{C}^{-1} \dot{\boldsymbol{E}} \boldsymbol{C}^{-1} \dot{\boldsymbol{E}}\right)$; and four coupled invariants: $\operatorname{tr}(\boldsymbol{S} \dot{\boldsymbol{E}}), \operatorname{tr}(\boldsymbol{S} \dot{\boldsymbol{E}} \boldsymbol{S C}), \operatorname{tr}\left(\boldsymbol{S} \dot{\boldsymbol{E}} \boldsymbol{C}^{-1} \dot{\boldsymbol{E}}\right)$, and $\operatorname{tr}(\boldsymbol{S} \dot{\boldsymbol{E}} \boldsymbol{S} \dot{\boldsymbol{E}})$. Any instance of GreEN's strain rate $\dot{\boldsymbol{E}}$ in the above invariants can be replaced by LoDGE's strain rate $\dot{\boldsymbol{E}}$ via their identity (15), while taking advantage of the property that $\operatorname{tr}(\boldsymbol{A} \boldsymbol{B})=\operatorname{tr}(\boldsymbol{B} \boldsymbol{A})$ for any $\boldsymbol{A}$ and $\boldsymbol{B}$.

From this set of allowable invariants, and the physical restrictions that are imposed on $\Phi$ by Fung's law and Truesdell's definition for hypo-elastic solids, one finds that the most general expression for such a potential is:

$$
\begin{aligned}
\Phi=\frac{1}{2}( & \lambda+\omega \operatorname{tr}(\boldsymbol{S C})) \operatorname{tr}\left(\boldsymbol{C}^{-1} \dot{\boldsymbol{E}}\right) \operatorname{tr}(\dot{\boldsymbol{E}} \boldsymbol{C}) \\
+ & \frac{1}{2}(2 \mu+\delta \operatorname{tr}(\boldsymbol{S C})) \operatorname{tr}(\dot{\boldsymbol{E}} \dot{\boldsymbol{E}}) \\
+ & \frac{1}{2} \alpha \operatorname{tr}(\boldsymbol{S} \dot{\boldsymbol{E}}) \operatorname{tr}(\dot{\boldsymbol{E}} \boldsymbol{C}) \\
& +(\beta-1) \operatorname{tr}(\boldsymbol{S} \dot{\boldsymbol{E}} \dot{\boldsymbol{E}} \boldsymbol{C}),
\end{aligned}
$$

where $\alpha, \beta, \delta, \lambda, \mu$, and $\omega$ are the material constants, with $\alpha, \beta, \delta$, and $\omega$ being dimensionless, while $\lambda$ and $\mu$ have units of stress; specifically, of force per unit undeformed area, like $S$. For incompressible materials, only $\beta, \delta$, and $\mu$ survive [13].

It is worth pointing out that all contractions are over contravariant/covariant index pairs, as in general tensor analysis $[34,46]$. This is an implicit characteristic of Lagrangian constructions built upon Cartesian frames. This characteristic becomes somewhat blurred whenever Cartesian frames are adopted in an Eulerian setting. This is another contributing factor for why this author constructs his theories in terms of Lagrangian fields, instead of Eulerian fields.

\subsection{Tangent Modulus}

Taking the potential function in Eq. (28), taking its gradients according to Eq. (26), and then pushing this result forward into the Eulerian frame according to Eqs. $(12,13,17$ \& 27) leads to (after much algebra) the following elegant form

$$
\begin{aligned}
\oplus= & \left(\lambda|\mathbf{F}|^{-1}+\omega \operatorname{tr}(\boldsymbol{\sigma})\right) \mathbf{I} \otimes \mathbf{I} \\
& +\left(2 \mu|\mathbf{F}|^{-1}+\delta \operatorname{tr}(\boldsymbol{\sigma})\right) \mathbf{I} \odot \mathbf{I} \\
& +\alpha \frac{1}{2}(\boldsymbol{\sigma} \otimes \mathbf{I}+\mathbf{I} \otimes \boldsymbol{\sigma}) \\
& +2(\beta-1) \frac{1}{2}(\boldsymbol{\sigma} \odot \mathbf{I}+\mathbf{I} \odot \boldsymbol{\sigma}),
\end{aligned}
$$

where $\otimes$ and $\odot$ are the outer- and inner-dyadic products, respectively, whose symmetric components are defined by

$$
\begin{aligned}
{\left[\frac{1}{2}(\boldsymbol{A} \otimes \boldsymbol{B}\right.} & +\boldsymbol{B} \otimes \boldsymbol{A})]_{i j k \ell} \\
& =\frac{1}{2}\left(\mathrm{~A}_{i j} \mathrm{~B}_{k \ell}+\mathrm{B}_{i j} \mathrm{~A}_{k \ell}\right),
\end{aligned}
$$

and by

$$
\begin{aligned}
{\left[\frac{1}{2}(\boldsymbol{A} \odot \boldsymbol{B}+\boldsymbol{B} \odot \boldsymbol{A})\right]_{i j k \ell}=} \\
\frac{1}{4}\left(\mathrm{~A}_{i k} \mathrm{~B}_{j \ell}+\mathrm{A}_{i \ell} \mathrm{B}_{j k}\right. \\
\left.+\mathrm{B}_{i k} \mathrm{~A}_{j \ell}+\mathrm{B}_{i \ell} \mathrm{A}_{j k}\right),
\end{aligned}
$$

where it is supposed that $\boldsymbol{A}$ and $\boldsymbol{B}$ are symmetric. These dyadic products possess both minor, i.e., $\theta_{i j k \ell}=\theta_{j i k \ell}=\theta_{i j \ell k}=\theta_{j i \ell k}$, and major, viz., $\theta_{i j k \ell}=\theta_{k \ell i j}$, symmetries.

$\$$. Previously, the author denoted $\delta$ as $\alpha$. The -1 term in $\beta-1$ is introduced so an outcome of this potential reproduces FunG's law in simple extension. 
Recall the $\lambda$ and $\mu$ have units of force per unit undeformed area, so that when divided by $|\mathbf{F}|$, one acquires units of force per unit deformed area, just like CAUCHY stress $\sigma$, and therefore, all terms in Eq. (29) have the same physical dimensions.

\subsection{Model for Parenchyma}

In the author's prior studies of the incompressible version of this material [13], he found that the $\beta$ and $\delta$ terms in the model apply to two, different, material classes. The $\delta$ term has application for tissues that predominantly carry compressive loads, while the $\beta$ term has application for tissues that predominantly carry tensile loads. This result follows from an analysis based on HILL's stability criterion [23, 24]. The author expects, but has not yet proven, that the same will likely be true for the $\alpha$ and $\omega$ terms. As such, because the alveoli are tension-only structures, a reasonable initial simplification of the above model would be to neglect these two compression-dominant terms, viz., $\delta$ and $\omega$, leaving

$$
\begin{gathered}
\oplus=\lambda|\mathbf{F}|^{-1} \mathbf{I} \otimes \mathbf{I}+\alpha \frac{1}{2}(\boldsymbol{\sigma} \otimes \mathbf{I}+\mathbf{I} \otimes \boldsymbol{\sigma})+ \\
2 \mu|\mathbf{F}|^{-1} \mathbf{I} \odot \mathbf{I}+2(\beta-1) \frac{1}{2}(\boldsymbol{\sigma} \odot \mathbf{I}+\mathbf{I} \odot \boldsymbol{\sigma}) .
\end{gathered}
$$

We now have a simple continuum model in which to seek a representation for parenchyma.

\section{Alternative Form}

It turns out that working with the CAUCHY stress $\sigma$ is complicated somewhat by the fact that it is a relative tensor field. A simpler formulation of the theory follows if one selects a different measure of stress to use in the Eulerian frame; in particular, the KIRCHHOFF stress $s$ defined by

$$
\boldsymbol{s}=|\mathbf{F}| \sigma
$$

relates to the second Piola-KIRchHoff stress via

$$
\boldsymbol{S}=|\mathbf{F}| \mathbf{F}^{-1} \sigma \mathbf{F}^{-T}=\mathbf{F}^{-1} s \mathbf{F}^{-T},
$$

whose rates therefore obey a field transfer of

$$
\dot{\boldsymbol{S}}=|\mathbf{F}| \mathbf{F}^{-1} \stackrel{\circ}{\sigma} \mathbf{F}^{-T}=\mathbf{F}^{-1} \stackrel{\Delta}{\boldsymbol{S}} \mathbf{F}^{-T},
$$

and therefore one arrives at the identity

$$
\stackrel{\Delta}{s}=|\mathbf{F}| \stackrel{\circ}{\sigma}
$$

wherein $\stackrel{\Delta}{s}=\mathrm{D} \boldsymbol{s} / \mathrm{D} t-\boldsymbol{l} \boldsymbol{s}-\boldsymbol{s} \boldsymbol{l}^{T}$ is the upperconvected derivative of OLDROYD [38].

With this change in variable in place, our hypo-elastic theory takes on a simpler form of

$$
\dot{\boldsymbol{S}}=\mathbb{M}: \dot{\boldsymbol{\varepsilon}} \quad \text { or } \quad \stackrel{\Delta}{\boldsymbol{s}}=\mathbb{m}: \boldsymbol{d},
$$

whose tangent moduli are defined by

$$
\mathbb{M} \equiv \Theta \quad \text { and } \quad \mathbb{m}=|\mathbf{F}| \oplus,
$$

with their components mapping as

$$
\begin{aligned}
\mathrm{m}_{i j k \ell} & =\mathrm{F}_{i I} \mathrm{~F}_{j J} \mathrm{M}_{I J K L} \mathrm{~F}_{K k}^{-1} \mathrm{~F}_{L \ell}^{-1}, \\
\mathrm{M}_{I J K L} & =\mathrm{F}_{I i}^{-1} \mathrm{~F}_{J j}^{-1} \mathrm{~m}_{i j k \ell} \mathrm{F}_{k K} \mathrm{~F}_{\ell L},
\end{aligned}
$$

which is an absolute variant of Eq. (27). The tangent modulus written in Eq. (32) has an equivalent representation of

$$
\begin{aligned}
& m=\lambda \mathbf{I} \otimes \mathbf{I}+\alpha \frac{1}{2}(\boldsymbol{s} \otimes \mathbf{I}+\mathbf{I} \otimes \mathbf{s})+ \\
& 2 \mu \mathbf{I} \odot \mathbf{I}+2(\beta-1) \frac{1}{2}(\boldsymbol{s} \odot \mathbf{I}+\mathbf{I} \odot \boldsymbol{s}) .
\end{aligned}
$$

whenever the KIRCHHOFF stress is used instead of the CaUchy stress.

\subsection{Tensor to Matrix Maps}

With the modulus being a forth-order tensor, it is convenient to map the tensorial constitutive equation (37) into a VoIGT matrix equivalent [2]. There are several VoIgT notations in use, but the 


\begin{tabular}{|l|c|c|c|c|}
\hline Notation & \multicolumn{4}{|c|}{ Index } \\
\hline Tensor & 11 & 22 & 33 & 12,21 \\
VoIGT & 1 & 2 & 3 & 4 \\
\hline
\end{tabular}

Table 1: Mappings between tensor and VoIGT indices for planar deformations of compressible media. Tensor indices 13, 23, 31, and 32 associate with zero valued elements.

NAdeau \& Ferrari [36] scheme is the only one where the mappings are frame invariant, at least of those that this author is aware of, and as such, their's is the VoIGT notation of choice.

VoIGT notations establish a mapping between the indices of a symmetric tensor and an alternative array representation. For general analysis, VoIGT arrays are of dimension six (cf. Nadeau \& Ferrari [36]). For planar deformations of incompressible materials, VoIGT arrays can reduce down to a dimension of three (the 13 and 23 out-of-plane shear components are taken to be zero, while the 33 component is governed by the incompressibility constraint, cf. FREED [13, App. 3]). For planar deformations of compressible materials, of which the experiments that follow are special cases thereof, VoIGT arrays reduce down to a dimension of four, with index mappings specified according to Table 1.

A symmetric second-order tensor, say $\boldsymbol{T}$, subject to some planar deformation will have a VoIGT notation of

$$
\mathrm{T}_{\xi}=\left\{\begin{array}{llll}
\mathrm{T}_{1} & \mathrm{~T}_{2} & \mathrm{~T}_{3} & \mathrm{~T}_{4}
\end{array}\right\}^{T},
$$

where the VoIGT components $T_{\xi}$ relate to their tensor components $\mathrm{T}_{i j}$ via the mappings given in Table 1. By convention, Latin indices are used to denote tensor components, while Greek indices are used to denote VoIGT components.

The Nadeau-Ferrari scheme differs from other VoIGT notations in that their mapping applies to all symmetric tensors of second order, both kinematic and kinetic. This feature is a direct consequence of their introduction of a diagonal ReUTER matrix (cf. with their App. B)

$$
\Gamma_{\xi \zeta}=\left\lceil\begin{array}{llll}
1 & 1 & 1 & 2
\end{array}\right. \text {, }
$$

which arises whenever contractions occur between VoIgT arrays and/or matrices [36].

The VoIGT notation for a forth-order tensor with minor symmetry, e.g., m, is a matrix, i.e.,

$$
\mathrm{m}_{\xi \zeta}=\left[\begin{array}{llll}
\mathrm{m}_{11} & \mathrm{~m}_{12} & \mathrm{~m}_{13} & \mathrm{~m}_{14} \\
\mathrm{~m}_{21} & \mathrm{~m}_{22} & \mathrm{~m}_{23} & \mathrm{~m}_{24} \\
\mathrm{~m}_{31} & \mathrm{~m}_{32} & \mathrm{~m}_{33} & \mathrm{~m}_{34} \\
\mathrm{~m}_{41} & \mathrm{~m}_{42} & \mathrm{~m}_{43} & \mathrm{~m}_{44}
\end{array}\right],
$$

where, e.g., VoIGT component $\mathrm{m}_{43}$ associates with tensor component $\mathrm{m}_{1233}$, in accordance with the mappings specified in Table 1 . In general, this matrix is not symmetric. If it happens to be symmetric, then $m$ is said to possess major symmetry (in addition to its minor symmetry).

VoIGT components for the two dyadic products provided in Eqs. (30 \& 31) can be found in App. 4 of Freed, Einstein \& Vesely [15] for the general case of six-dimensional arrays. Below, they are given for the special case of planar deformations obeying the maps in Table 1 . These operators yield tensors that possess both minor and major symmetry; therefore, their VoIGT matrix representations are symmetric, regardless of Voigt dimension.

The outer product $\mathbb{C}=\frac{1}{2}(\boldsymbol{A} \otimes \boldsymbol{B}+\boldsymbol{B} \otimes \boldsymbol{A})$ has components quantified by

$$
\begin{aligned}
& C_{11}=A_{1} B_{1}, \\
& C_{12}=\frac{1}{2}\left(A_{1} B_{2}+A_{2} B_{1}\right), \\
& C_{13}=\frac{1}{2}\left(A_{1} B_{3}+A_{3} B_{1}\right), \\
& C_{14}=\frac{1}{2}\left(A_{1} B_{4}+A_{4} B_{1}\right), \\
& C_{22}=A_{2} B_{2}, \\
& C_{23}=\frac{1}{2}\left(A_{2} B_{3}+A_{3} B_{2}\right), \\
& C_{24}=\frac{1}{2}\left(A_{2} B_{4}+A_{4} B_{2}\right), \\
& C_{33}=A_{3} B_{3}, \\
& C_{34}=\frac{1}{2}\left(A_{3} B_{4}+A_{4} B_{3}\right), \\
& C_{44}=A_{4} B_{4},
\end{aligned}
$$


while inner product $\mathbb{C}=\frac{1}{2}(\boldsymbol{A} \odot \boldsymbol{B}+\boldsymbol{B} \odot \boldsymbol{A})$ has components quantified by

$$
\begin{aligned}
& C_{11}=A_{1} B_{1}, \\
& C_{12}=A_{4} B_{4}, \\
& C_{13}=0, \\
& C_{14}=\frac{1}{2}\left(A_{1} B_{4}+A_{4} B_{1}\right), \\
& C_{22}=A_{2} B_{2}, \\
& C_{23}=0 \\
& C_{24}=\frac{1}{2}\left(A_{2} B_{4}+A_{4} B_{2}\right), \\
& C_{33}=A_{3} B_{3}, \\
& C_{34}=\frac{1}{2}\left(A_{3} B_{4}+A_{4} B_{3}\right), \\
& C_{44}=\frac{1}{4}\left(A_{1} B_{2}+A_{2} B_{1}+2 A_{4} B_{4}\right),
\end{aligned}
$$

with symmetry supplying the remaining elements of both $\mathrm{C}_{\xi \zeta}$ VoIGT arrays.

The hypo-elastic constitutive model (37), when expressed in the Eulerian frame, has a NADEAU-FERRARI form of

$$
{\stackrel{\Delta}{\mathrm{s}_{\xi}}}=\mathrm{m}_{\xi \zeta} \Gamma_{\zeta \eta} \mathrm{d}_{\eta}
$$

when expressed in VoIGT component notation.

\section{Experiments}

To gain insight into the behavior of hypo-elastic material model (37) defined by tangent modulus (40), the ordinary differential equations (ODEs) that arise from several boundary-value problems (BVPs) used for material characterization are constructed. Each assumes a material response that is isotropic in stress and strain rate.

The tangent modulus in Eq. (40) is comprised of four separate moduli; specifically,

$$
m={ }^{\lambda} m+{ }_{m} m_{m}+{ }^{\alpha} m+{ }^{\beta} m,
$$

wherein

$$
\lambda_{m}=\lambda \mathbf{I} \otimes \mathbf{I},
$$

and

$$
\mu_{m n}=2 \mu \mathbf{I} \odot \mathbf{I}
$$

with $\lambda$ and $\mu$ being the two LAMÉ constants ${ }^{\S}$, whereas

$$
\alpha_{m n}=\alpha \frac{1}{2}(s \otimes \mathbf{I}+\mathbf{I} \otimes s),
$$

and

$$
\beta_{m n}=2(\beta-1) \frac{1}{2}(s \odot \mathbf{I}+\mathbf{I} \odot \mathbf{s}),
$$

with $\alpha$ and $\beta$ being constants that are in the spirit of FUNG's $\beta$ parameter in his law (24).

In VoIGT notation, the two LAMÉ moduli $\lambda_{\text {m }}$ and $\mu_{\text {m }}$ are constant valued. In 4D notation, they are

$$
\lambda_{\xi \zeta}=\lambda\left[\begin{array}{llll}
1 & 1 & 1 & 0 \\
1 & 1 & 1 & 0 \\
1 & 1 & 1 & 0 \\
0 & 0 & 0 & 0
\end{array}\right],
$$

and

$$
\mu_{\mathrm{m}_{\xi \zeta}}=2 \mu\left[\begin{array}{cccc}
1 & 0 & 0 & 0 \\
0 & 1 & 0 & 0 \\
0 & 0 & 1 & 0 \\
0 & 0 & 0 & 1 / 2
\end{array}\right],
$$

while the two FuNG moduli ${ }^{\alpha}$ m and ${ }^{\beta}$ mn have components that will depend upon the BVP being addressed.

VoIGT notations for the tangent moduli ${ }^{\alpha} \mathrm{m}_{\xi \xi}$ and ${ }^{\beta} \mathrm{m}_{\xi \xi}$, and the strain-rate vector $\Gamma_{\xi \zeta} d_{\zeta}$, are now quantified for several example BVPs. The result is a different algebraic expression describing stress rate $\Delta_{\xi}$ for each experiment. It is through an understanding of these tangent moduli (48-51), and how they vary for different BVPs, that one can begin to acquire an intuition about this material model and its behavior.

A means by which one would quantify the KIRCHHOFF stress components $s_{\xi}$ via experiment is also provided, so that theory and experiment can be compared.

$\S$. Truesdell's [53] hypo-elastic body of grade zero (his simplest hypo-elastic solid) has a tangent modulus with $\lambda_{\oplus}=\lambda \mathbf{I} \otimes \mathbf{I}$ and $\mu_{\oplus}=2 \mu \mathbf{I} \odot \mathbf{I}$. His theory has elastic LAMÉ constants $\lambda$ and $\mu$ with dimensions of force per unit deformed area; whereas, our theory has Lamé constants $\lambda$ and $\mu$ with dimensions of force per unit undeformed area. 


\subsection{Uniaxial Extension}

This experiment is described by a deformation gradient in $\mathbb{R}^{3}$ of

$$
\mathrm{F}_{i J}=\left[\begin{array}{ccc}
\lambda_{\ell} & 0 & 0 \\
0 & \lambda_{w} & 0 \\
0 & 0 & \lambda_{w}
\end{array}\right],
$$

where $\lambda_{\ell}$ is the stretch applied to the sample, while $\lambda_{w}$ is its responding transverse stretch.

The nominal force required to impose this stretch, when expressed in terms of the first PiolaKirchhoff stress $\mathbf{P}$ in $\mathbb{R}^{3}$, is simply

$$
\mathrm{P}_{i J}=\left[\begin{array}{lll}
\mathrm{\top} & 0 & 0 \\
0 & 0 & 0 \\
0 & 0 & 0
\end{array}\right],
$$

where $\mathbf{P}=|\mathbf{F}| \sigma \mathbf{F}^{-T}=s \mathbf{F}^{-T}$ with $\mathrm{T}=$ $f / A_{0}$ being the engineering stress, $f$ the applied force, and $A_{0}$ the initial cross-sectional area that force $f$ is acting across. From these two fields, all others can be determined.

For this BVP, a strain rate of

$$
\Gamma_{\xi \zeta} \mathrm{d}_{\zeta}=\left\{\begin{array}{c}
\dot{\lambda}_{\ell} / \lambda_{\ell} \\
\dot{\lambda}_{w} / \lambda_{w} \\
\dot{\lambda}_{w} / \lambda_{w} \\
0
\end{array}\right\}
$$

is imposed, producing a stress response of

$$
\mathrm{s}_{\xi}=\left\{\begin{array}{l}
\mathrm{s} \\
0 \\
0 \\
0
\end{array}\right\}=\left\{\begin{array}{c}
\lambda_{\ell} \mathrm{T} \\
0 \\
0 \\
0
\end{array}\right\}
$$

whose LIE rate-of-change is

$$
\stackrel{\Delta}{s}_{\xi}=\left\{\begin{array}{c}
\dot{\mathrm{s}}-2 \mathrm{~s} \dot{\lambda}_{\ell} / \lambda_{\ell} \\
0 \\
0 \\
0
\end{array}\right\}
$$

where $\stackrel{\Delta}{s}=\dot{s}-\boldsymbol{l} \boldsymbol{s}-\boldsymbol{s} \boldsymbol{l}^{T}$. Because the deformation is considered to be homogeneous, term $\operatorname{grad}(\boldsymbol{s}) \cdot v$ in $\mathrm{D} \boldsymbol{s} / \mathrm{D} t$ does not contribute to the overall accumulation of stress.

The two, Fung, tangent moduli are given by

$$
{ }^{\alpha} \mathrm{m}_{\xi \zeta}=\alpha\left[\begin{array}{cccc}
\mathrm{s} & \mathrm{s} / 2 & \mathrm{~s} / 2 & 0 \\
\mathrm{~s} / 2 & 0 & 0 & 0 \\
\mathrm{~s} / 2 & 0 & 0 & 0 \\
0 & 0 & 0 & 0
\end{array}\right],
$$

and

$$
{ }^{\beta} \mathrm{m}_{\xi \zeta}=2(\beta-1)\left[\begin{array}{cccc}
\mathrm{s} & 0 & 0 & 0 \\
0 & 0 & 0 & 0 \\
0 & 0 & 0 & 0 \\
0 & 0 & 0 & \mathrm{~s} / 4
\end{array}\right],
$$

where $s$ is the 11-component of the КіRcнноғF stress.

\subsection{Equi-Biaxial Extension}

This experiment is described by a deformation gradient of

$$
\mathrm{F}_{i J}=\left[\begin{array}{ccc}
\lambda_{\ell} & 0 & 0 \\
0 & \lambda_{\ell} & 0 \\
0 & 0 & \lambda_{w}
\end{array}\right]
$$

and a first Piola-KIRChHoff stress of

$$
\mathrm{P}_{i J}=\left[\begin{array}{ccc}
\mathrm{T} & 0 & 0 \\
0 & \mathrm{~T} & 0 \\
0 & 0 & 0
\end{array}\right]
$$

both being expressed in $\mathbb{R}^{3}$. From these two fields, all others can be determined.

For this BVP, a strain rate of

$$
\Gamma_{\xi \zeta} \mathrm{d}_{\zeta}=\left\{\begin{array}{c}
\dot{\lambda}_{\ell} / \lambda_{\ell} \\
\dot{\lambda}_{\ell} / \lambda_{\ell} \\
\dot{\lambda}_{w} / \lambda_{w} \\
0
\end{array}\right\}
$$

is imposed, causing a stress response of

$$
\mathrm{s}_{\xi}=\left\{\begin{array}{l}
\mathrm{s} \\
\mathrm{s} \\
0 \\
0
\end{array}\right\}=\left\{\begin{array}{c}
\lambda_{\ell} T \\
\lambda_{\ell} T \\
0 \\
0
\end{array}\right\}
$$


with its LIE rate-of-change being

$$
\dot{\Delta}_{\xi}=\left\{\begin{array}{c}
\dot{\mathrm{s}}-2 \mathrm{~s} \dot{\lambda}_{\ell} / \lambda_{\ell} \\
\dot{\mathrm{s}}-2 \mathrm{~s} \dot{\lambda}_{\ell} / \lambda_{\ell} \\
0 \\
0
\end{array}\right\}
$$

where, again, the deformation is considered to be homogeneous.

The two, Fung, tangent moduli are given by

$$
{ }^{\alpha} \mathrm{m}_{\xi \zeta}=\alpha\left[\begin{array}{cccc}
\mathrm{s} & \mathrm{s} & \mathrm{s} / 2 & 0 \\
\mathrm{~s} & \mathrm{~s} & \mathrm{~s} / 2 & 0 \\
\mathrm{~s} / 2 & \mathrm{~s} / 2 & 0 & 0 \\
0 & 0 & 0 & 0
\end{array}\right],
$$

and

$$
{ }^{\beta} \mathrm{m}_{\xi \zeta}=2(\beta-1)\left[\begin{array}{cccc}
\mathrm{s} & 0 & 0 & 0 \\
0 & \mathrm{~s} & 0 & 0 \\
0 & 0 & 0 & 0 \\
0 & 0 & 0 & \mathrm{~s} / 2
\end{array}\right],
$$

which are fuller than their uniaxial counterparts.

\subsection{Inflation}

This experiment mimics the predominant mechanisms by which lung deforms. It is described by a deformation gradient with components

$$
\mathrm{F}_{i J}=\left[\begin{array}{ccc}
\lambda_{v} & 0 & 0 \\
0 & \lambda_{v} & 0 \\
0 & 0 & \lambda_{v}
\end{array}\right],
$$

and a first Piola-KIRCHHOFF stress of

$$
\mathrm{P}_{i J}=\left[\begin{array}{ccc}
\mathrm{T} & 0 & 0 \\
0 & \mathrm{~T} & 0 \\
0 & 0 & \mathrm{~T}
\end{array}\right],
$$

both being expressed in $\mathbb{R}^{3}$. Hydrostatic pressure is defined by $p=-\frac{1}{3} \operatorname{tr}(\sigma)=-\mathrm{T} / \lambda_{v}^{2}$. From these fields, all others can be determined.

For this BVP, a strain rate of

$$
\Gamma_{\xi \zeta} \mathrm{d}_{\zeta}=\left\{\begin{array}{c}
\dot{\lambda}_{v} / \lambda_{v} \\
\dot{\lambda}_{v} / \lambda_{v} \\
\dot{\lambda}_{v} / \lambda_{v} \\
0
\end{array}\right\}
$$

is imposed, whose stress response is

$$
\mathrm{s}_{\xi}=\left\{\begin{array}{l}
\mathrm{s} \\
\mathrm{s} \\
\mathrm{s} \\
0
\end{array}\right\}=\left\{\begin{array}{c}
\lambda_{v} \top \\
\lambda_{v} \mathrm{~T} \\
\lambda_{v} \mathrm{~T} \\
0
\end{array}\right\}
$$

so that $p=-\mathrm{s} / \lambda_{v}^{3}$, with its LIE rate-of-change being given by

$$
\overrightarrow{\mathrm{s}}_{\xi}=\left\{\begin{array}{c}
\dot{\mathrm{s}}-2 \mathrm{~s} \dot{\lambda}_{v} / \lambda_{v} \\
\dot{\mathrm{s}}-2 \mathrm{~s} \dot{\lambda}_{v} / \lambda_{v} \\
\dot{\mathrm{s}}-2 \mathrm{~s} \dot{\lambda}_{v} / \lambda_{v} \\
0
\end{array}\right\}
$$

where a homogeneous deformation is assumed.

The two, Fung, tangent moduli are given by

$$
\alpha_{\mathrm{m}_{\xi \zeta}}=\alpha\left[\begin{array}{cccc}
\mathrm{s} & \mathrm{s} & \mathrm{s} & 0 \\
\mathrm{~s} & \mathrm{~s} & \mathrm{~s} & 0 \\
\mathrm{~s} & \mathrm{~s} & \mathrm{~s} & 0 \\
0 & 0 & 0 & 0
\end{array}\right]
$$

and

$$
{ }^{\beta} \mathrm{m}_{\xi \zeta}=2(\beta-1)\left[\begin{array}{cccc}
\mathrm{s} & 0 & 0 & 0 \\
0 & \mathrm{~s} & 0 & 0 \\
0 & 0 & \mathrm{~s} & 0 \\
0 & 0 & 0 & \mathrm{~s} / 2
\end{array}\right] .
$$

It is here, in the BVP defining inflation, that the resemblance between ${ }^{\lambda}$ m and ${ }^{\alpha}$ mn, and between $\mu_{m n}$ and ${ }^{\beta}$ mn, becomes most evident.

\subsection{Simple Shear}

This experiment is described by a deformation gradient in $\mathbb{R}^{3}$ of

$$
\mathrm{F}_{i J}=\left[\begin{array}{ccc}
1 & \gamma & 0 \\
0 & 1 & 0 \\
0 & 0 & \lambda_{w}
\end{array}\right],
$$

where $\gamma$ is the magnitude of shear, while $\gamma / 2$ is often referred to as the shear strain. Stretch $\lambda_{w}$ arises as a response to an imposed plane-stress condition. 
The stress state in concert with shear, when expressed in terms of the first Piola-KirchHoff stress $\mathbf{P}$ in $\mathbb{R}^{3}$, is described by [33]

$\mathbf{P}_{i J}=\left[\begin{array}{ccc}\mathrm{P}_{11} & \mathrm{P}_{12} & 0 \\ \mathrm{P}_{21} & \mathrm{P}_{22} & 0 \\ 0 & 0 & \mathrm{P}_{33}\end{array}\right], \quad \mathrm{P}_{21}=\mathrm{P}_{12}-\gamma \mathrm{P}_{22}$.

Two of these tractions can be experimentally determined in rectilinear shear. They are the shear stress $\mathrm{P}_{12}=f_{1} / A_{0}$ and the normal stress $\mathrm{P}_{22}=f_{2} / A_{0}$, where $A_{0}$ is the initial area of the surface of shearing. Force $f_{1}$ is applied in the shearing direction, while $f_{2}$ is the reaction force that arises normal to the shear plane.

Components $P_{11}$ and $P_{33}$ cannot be determined in a rectilinear simple-shear experiment. The $\mathrm{P}_{11}$ normal stress is carried internally by the grips (it can, however, be determined in curvilinear shear experiments). The $\mathrm{P}_{33}$ normal stress will be zero whenever one shears planar membranes. This is because of the plane-stress assumption, which is the situation considered below.

From the two tensor fields given above, all others can be determined. For this BVP, VoIGT components of the ReuTER, adjusted, strain rate are

$$
\Gamma_{\xi \zeta} \mathrm{d}_{\zeta}=\left\{\begin{array}{c}
0 \\
0 \\
\dot{\lambda}_{w} / \lambda_{w} \\
\dot{\gamma}
\end{array}\right\}
$$

while the experimental stress response, assuming plane stress, has components

$$
\mathrm{s}_{\xi}=\left\{\begin{array}{c}
\mathrm{s}_{1} \\
\mathrm{~s}_{2} \\
0 \\
\mathrm{~s}_{\tau}
\end{array}\right\}=\left\{\begin{array}{c}
\mathrm{P}_{11}+\gamma f_{1} / A_{0} \\
f_{2} / A_{0} \\
\mathrm{P}_{33}=0 \\
f_{1} / A_{0}
\end{array}\right\},
$$

with its LIE rate-of-change being governed by

$$
\stackrel{\Delta}{s}_{\xi}=\left\{\begin{array}{c}
\dot{s}_{1}-2 \mathrm{~s}_{\tau} \dot{\gamma} \\
\dot{s}_{2} \\
0 \\
\dot{\mathrm{s}}_{\tau}-\mathrm{s}_{2} \dot{\gamma}
\end{array}\right\}
$$

where the deformation is once again taken to be homogeneous.

The two Fung tangent moduli are

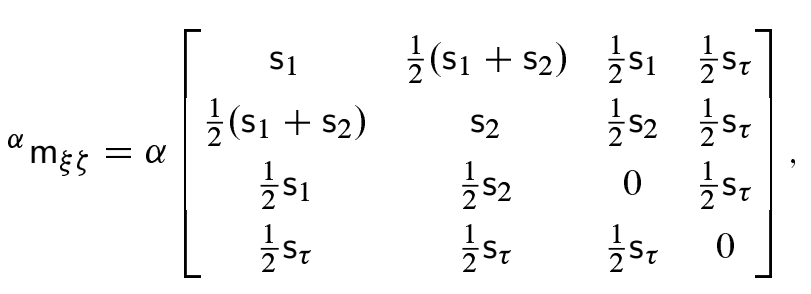

and

$$
\beta_{\mathrm{m}_{\xi \zeta}}=2(\beta-1)\left[\begin{array}{cccc}
\mathrm{s}_{1} & 0 & 0 & \frac{1}{2} \mathrm{~s}_{\tau} \\
0 & \mathrm{~s}_{2} & 0 & \frac{1}{2} \mathrm{~s}_{\tau} \\
0 & 0 & 0 & \frac{1}{2} \mathrm{~s}_{\tau} \\
\frac{1}{2} \mathrm{~s}_{\tau} & \frac{1}{2} \mathrm{~s}_{\tau} & \frac{1}{2} \mathrm{~s}_{\tau} & \frac{1}{4}\left(\mathrm{~s}_{1}+\mathrm{s}_{2}\right)
\end{array}\right],
$$

where tangent moduli $\alpha_{m n}$ and $\beta_{m m}$ are seen to be vastly different from those of the prior deformation histories, which are all shear-free in the sense of LodGe [34].

\subsection{Inflation/Compression}

Uniaxial extension, although a common experiment for the purpose of material characterization for most materials, is not a viable experiment for characterizing parenchyma. To study the uniaxial response of spongy lung, LaI-FooK et al. [30] inflated lobes to a preset pressure, and then compressed these lobes between a pair of parallel platens while holding the alveolar pressure constant during the compression loading cycle. This is a significantly different experiment from those just discussed; it has a history effect.

We use the pressure/volume response just derived as an intermediate reference state, to which a perturbation in deformation is imposed as described by

$$
\mathrm{F}_{i J}=\left[\begin{array}{ccc}
\lambda_{\ell} & 0 & 0 \\
0 & \lambda_{w} & 0 \\
0 & 0 & \lambda_{w}
\end{array}\right]
$$


where now

$$
\begin{array}{ll}
\lambda_{\ell}=\frac{\ell(t)}{\ell_{0}}=\lambda_{\ell}^{\Delta} \lambda_{v}, & \lambda_{\ell}^{\Delta}=\frac{\ell(t)}{\ell_{v}}, \\
\lambda_{w}=\frac{w(t)}{w_{0}}=\lambda_{w}^{\Delta} \lambda_{v}, & \lambda_{w}^{\Delta}=\frac{w(t)}{w_{v}}, \\
\lambda_{v}=\frac{\ell_{v}}{\ell_{0}} \equiv \frac{w_{v}}{w_{0}}, &
\end{array}
$$

with $\lambda_{v}$ being held constant; consequently,

$$
\dot{\mathrm{F}}_{i J}=\left[\begin{array}{ccc}
\dot{\lambda}_{\ell}^{\Delta} \lambda_{v} & 0 & 0 \\
0 & \dot{\lambda}_{w}^{\Delta} \lambda_{v} & 0 \\
0 & 0 & \dot{\lambda}_{w}^{\Delta} \lambda_{v}
\end{array}\right]
$$

Associated with this deformation one has components for the KIRCHHOFF stress that look like

$$
\mathrm{s}_{i j}=\left[\begin{array}{ccc}
\mathrm{s}_{p}-\mathrm{s}_{\Delta} & 0 & 0 \\
0 & \mathrm{~s}_{p}+\frac{1}{2} \mathrm{~s}_{\Delta} & 0 \\
0 & 0 & \mathrm{~s}_{p}+\frac{1}{2} \mathrm{~s}_{\Delta}
\end{array}\right],
$$

where $\mathrm{s}_{p}$ is the hydrostatic component of KIRchHOFF stress, which is given by Eq. (60), while $\mathrm{s}_{\Delta}=\lambda_{\ell} \mathrm{T}_{\Delta}=\lambda_{\ell}^{\Delta} \lambda_{v} \mathrm{~T}_{\Delta}$ where $\mathrm{T}_{\Delta}=$ $f_{\Delta} / A_{0}$ with $f_{\Delta}$ being the increment of compressive force that is applied. The trace of this stress state is constant, and therefore, the state of alveolar pressure remains constant over the loading perturbation. The above formulation assumes an isotropic material response.

With the above fields in hand, the Reuter modified strain rate is

$$
\Gamma_{\xi \zeta} \mathrm{d}_{\zeta}=\left\{\begin{array}{c}
\dot{\lambda}_{\ell}^{\Delta} / \lambda_{\ell}^{\Delta} \\
\dot{\lambda}_{w}^{\Delta} / \lambda_{w}^{\Delta} \\
\dot{\lambda} \Delta / \lambda_{w}^{\Delta} \\
0
\end{array}\right\}
$$

with a state of stress of

$$
\mathrm{s}_{\xi}=\left\{\begin{array}{c}
\mathrm{s}_{1} \\
\mathrm{~s}_{2} \\
\mathrm{~s}_{3} \\
0
\end{array}\right\}=\left\{\begin{array}{c}
\mathrm{s}_{p}-\mathrm{s}_{\Delta} \\
\mathrm{s}_{p}+\frac{1}{2} \mathrm{~s}_{\Delta} \\
\mathrm{s}_{p}+\frac{1}{2} \mathrm{~s}_{\Delta} \\
0
\end{array}\right\},
$$

whose LIE rate-of-change is

$$
\stackrel{\Delta}{s}_{\xi}=\left\{\begin{array}{c}
-\dot{\mathrm{s}}_{\Delta}-2\left(\mathrm{~s}_{p}-\mathrm{s}_{\Delta}\right) \dot{\lambda}_{\ell}^{\Delta} / \lambda_{\ell}^{\Delta} \\
\frac{1}{2} \dot{\mathrm{s}}_{\Delta}-\left(2 \mathrm{~s}_{p}+\mathrm{s}_{\Delta}\right) \dot{\lambda}_{w}^{\Delta} / \lambda_{w}^{\Delta} \\
\frac{1}{2} \dot{\mathrm{s}}_{\Delta}-\left(2 \mathrm{~s}_{p}+\mathrm{s}_{\Delta}\right) \dot{\lambda}_{w}^{\Delta} / \lambda_{w}^{\Delta} \\
0
\end{array}\right\},
$$

where a homogeneous deformation is assumed.

The two, Fung, tangent moduli are given by

$$
{ }^{\alpha} \mathrm{m}_{\xi \zeta}=\alpha\left[\begin{array}{cccc}
\mathrm{s}_{p}-\mathrm{s}_{\Delta} & \mathrm{s}_{p}-\frac{1}{4} \mathrm{~s}_{\Delta} & \mathrm{s}_{p}-\frac{1}{4} \mathrm{~s}_{\Delta} & 0 \\
\mathrm{~s}_{p}-\frac{1}{4} \mathrm{~s}_{\Delta} & \mathrm{s}_{p}+\frac{1}{2} \mathrm{~s}_{\Delta} & \mathrm{s}_{p}+\frac{1}{2} \mathrm{~s}_{\Delta} & 0 \\
\mathrm{~s}_{p}-\frac{1}{4} \mathrm{~s}_{\Delta} & \mathrm{s}_{p}+\frac{1}{2} \mathrm{~s}_{\Delta} & \mathrm{s}_{p}+\frac{1}{2} \mathrm{~s}_{\Delta} & 0 \\
0 & 0 & 0 & 0
\end{array}\right],
$$

and

$$
\begin{aligned}
& { }^{\beta_{\mathrm{m}_{\xi \xi}}}=2(\beta-1) \\
& \quad \times\left[\begin{array}{cccc}
\mathrm{s}_{p}-\mathrm{s}_{\Delta} & 0 & 0 & 0 \\
0 & \mathrm{~s}_{p}+\frac{1}{2} \mathrm{~s}_{\Delta} & 0 & 0 \\
0 & 0 & \mathrm{~s}_{p}+\frac{1}{2} \mathrm{~s}_{\Delta} & 0 \\
0 & 0 & 0 & \frac{1}{2} \mathrm{~s}_{p}-\frac{1}{8} \mathrm{~s}_{\Delta}
\end{array}\right] .
\end{aligned}
$$

\section{Model Response}

The previous section provides the general equations that govern a compressible hypo-elastic solid of the Fung type when subjected to a variety of BVPs that are commonly used for material characterization. This section discusses the response of this model to these BVPs in greater detail.

\subsection{Uniaxial}

In a uniaxial experiment, longitudinal stretch $\lambda_{\ell}$ is controlled to which stress $s=\lambda_{\ell} T$ and transverse (width) stretch $\lambda_{w}$ respond.

From the 22- or 33-components of the governing ODEs, one arrives at a PoIsson-like ratio being described by

$$
\tilde{v}=-\frac{\mathrm{d} \lambda_{w} / \lambda_{w}}{\mathrm{~d} \lambda_{\ell} / \lambda_{\ell}}=\frac{2 \lambda+\alpha \mathrm{s}}{4(\lambda+\mu)},
$$


that, in the neighborhood around zero stress, becomes the classic result $v=\lambda / 2(\lambda+\mu)$. The tilde in $\tilde{v}$ is used to denote that it is a tangent function, while $v$, without a tilde, retains its classic interpretation taken from the isotropic theory of linear elasticity [49]. Equation (52) allows for an additional linear dependence upon stress via parameter $\alpha$ in the transverse response. It is worth noting that $\tilde{v}$ does not depend upon the $\beta$ Fung parameter.

From the 11-component of the governing ODEs, an equivalent expression for Young's modulus is determined; it being,

$$
\begin{gathered}
\tilde{E}=\frac{\mathrm{ds}}{\mathrm{d} \lambda_{\ell} / \lambda_{\ell}}=\frac{\mu(3 \lambda+2 \mu)}{\lambda+\mu} \\
+\frac{\alpha \mu+2 \beta(\lambda+\mu)}{\lambda+\mu} \mathrm{s} \\
\quad-\frac{\alpha^{2} / 4}{\lambda+\mu} \mathrm{s}^{2},
\end{gathered}
$$

that, in the neighborhood of zero stress, simplifies to the classic expression for Young's modulus, viz., $E=\mu(3 \lambda+2 \mu) /(\lambda+\mu)$. Here $\mathrm{ds}=\mathrm{Td} \lambda_{\ell}+\lambda_{\ell} \mathrm{dT}$, where $\mathrm{dT}=\mathrm{d} f / A_{0}$.

Formula (53) is a Riccati differential equation with constant coefficients. It introduces a linear dependence upon stress via $\beta$, and a quadratic dependence upon stress through $\alpha$. To solve this ODE, we express it as

$$
\mathrm{s}^{\prime}=A \mathrm{~s}^{2}+B \mathrm{~s}+E,
$$

where $\mathrm{s}^{\prime}=\mathrm{ds} / \mathrm{d} \varepsilon$ with $\varepsilon=\ln \lambda_{\ell}$, while the coefficients have values of

$$
\begin{aligned}
& A=-\alpha^{2} / 4(\lambda+\mu), \\
& B=(\alpha \mu+2 \beta(\lambda+\mu)) /(\lambda+\mu), \\
& E=\mu(3 \lambda+2 \mu) /(\lambda+\mu),
\end{aligned}
$$

where $A<0, B>0$, and $E>0$.

Upon introducing a change in variable of $\mathrm{s}=-w^{\prime} / A w$ [41, pg. 84], one is lead to the well-known, second-order, linear, homogeneous ODE

$$
w^{\prime \prime}-B w^{\prime}+A E w=0 .
$$

Because $B^{2}-4 A E>0$, this equation for a damped oscillator has the solution [41, pg. 215]

$$
w(\varepsilon)=\mathrm{e}^{b \varepsilon}\left(C_{1} \mathrm{e}^{a \varepsilon}+C_{2} \mathrm{e}^{-a \varepsilon}\right),
$$

where $C_{1}$ and $C_{2}$ are constants of integration, with

$$
\begin{aligned}
& a=\frac{1}{2} \sqrt{B^{2}-4 A E}>0, \\
& b=\frac{1}{2} B>0,
\end{aligned}
$$

both of which are dimensionless.

From an initial condition (IC) of $s(0)=0$, one finds that

$$
C_{1}=\frac{a-b}{a+b} C_{2},
$$

from which it immediately follows that

$$
w(0)=\frac{2 a}{a+b} C_{2}=w_{0} \quad \& \quad w^{\prime}(0)=0 .
$$

Consequently,

$$
w=w_{0} \mathrm{e}^{b \varepsilon}\left(a \cosh (a \varepsilon)-\frac{b}{a} \sinh (a \varepsilon)\right) .
$$

One can now reconstruct $\mathrm{s}=-w^{\prime} / A w$, which gives

$$
\mathrm{s}=E \frac{\sinh (a \varepsilon)}{a \cosh (a \varepsilon)-b \sinh (a \varepsilon)},
$$

where $\mathrm{s}^{\prime}(0)=E$, as expected.

As shown in Fig. 3, this response is bounded from above and below by

$$
\begin{aligned}
\mathrm{s} & =\frac{E}{2 a}\left(\mathrm{e}^{2 a \varepsilon}-1\right) \quad \text { as } \quad b \rightarrow a, \\
\mathrm{~s} & =\frac{E}{a} \tanh (a \varepsilon) \quad \text { as } \quad b \rightarrow 0,
\end{aligned}
$$

with the response asymptoting to an ultimate strength of

$$
\mathrm{s} \asymp \frac{E}{a-b} \quad \text { as } \quad \varepsilon \rightarrow \infty,
$$

where $b$ is bound to the range $0 \leq b \leq a$. In actuality, most materials will fail long before they reach their asymptotic limit. Recall that $\varepsilon=\ln \lambda_{\ell}$ is the true strain. 
Bounds on Uniaxial Response

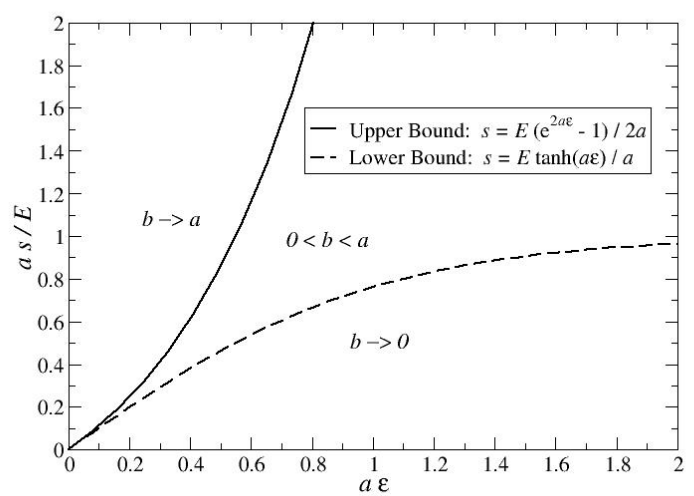

Figure 3: The domain of admissible stress/ strain responses in uniaxial extension. The bound from above is an exponential, while the bound from below is a hyperbolic tangent. Permissibility lies in between.

\subsection{Equi-Biaxial}

In the biaxial experiment, an equivalent pair of longitudinal stretches $\lambda_{\ell}$ are controlled to which an equivalent pair of stresses $\sigma$ and a transverse stretch $\lambda_{w}$ respond. The resulting ODEs can be combined into a single expression of

$$
\begin{aligned}
\frac{\mathrm{ds}}{\mathrm{d} \lambda_{\ell} / \lambda_{\ell}} & =\frac{2 \mu(3 \lambda+2 \mu)}{\lambda+2 \mu} \\
+ & \frac{4 \alpha \mu+2 \beta(\lambda+2 \mu)}{\lambda+2 \mu} \mathrm{s} \\
& -\frac{\alpha^{2} / 2}{\lambda+2 \mu} \mathrm{s}^{2} .
\end{aligned}
$$

This ODE can be solved by the same procedure used in the prior section for the uniaxial case.

In the absence of $\alpha$, e.g., for incompressible materials, uniaxial and equi-biaxial experiments are predicted to have the same stress dependence in their tangent moduli $\mathrm{ds} / \mathrm{d} \ln \lambda$, viz., $2 \beta$. This theoretical consequence has been experimentally verified [14].

\subsection{Inflation}

In an inflation or dilatation experiment, stretch $\lambda_{v}$ occurs uniformly in all directions to which a hydrostatic state of stress $s$ responds, as described by

$$
\mathrm{ds}=(3 \lambda+2 \mu+(3 \alpha+2 \beta) \mathrm{s}) \frac{\mathrm{d} \lambda_{v}}{\lambda_{v}},
$$

where $|\mathbf{F}|=\lambda_{v}^{3}$ so that $\mathrm{s}=|\mathbf{F}| \sigma=\lambda_{v} \mathrm{~T}$.

An effective bulk modulus can therefore be described by

$$
\tilde{\kappa}=\frac{\mathrm{ds}}{3 \mathrm{~d} \lambda_{v} / \lambda_{v}}=\lambda+\frac{2}{3} \mu+\left(\alpha+\frac{2}{3} \beta\right) \mathrm{s},
$$

whose units are force per unit undeformed area. This material parameter is of great importance in lung mechanics. The above result agrees with classical elasticity theory in the neighborhood of zero stress, viz., $\kappa=\lambda+\frac{2}{3} \mu$. Here an analogy between $\lambda$ and $\alpha$, and between $\mu$ and $\beta$, is strongly suggested.

Probably a more significant result follows from the fact that Eq. (58) can be integrated in closed form, producing the pressure-volume relationship

$$
\mathrm{s}=\frac{3 \lambda+2 \mu}{3 \alpha+2 \beta}\left(\left(\frac{v^{1 / 3}}{V^{1 / 3}}\right)^{3 \alpha+2 \beta}-1\right),
$$

or equivalently

$$
P=\frac{3 \lambda+2 \mu}{3 \alpha+2 \beta}\left(\left(\frac{v}{V}\right)^{\alpha+2 \beta / 3-1}-\frac{V}{v}\right),
$$

where $P=\mathrm{s} /|\mathbf{F}|$ is the alveolar pressure ${ }^{\text {If }}$ and $v$ is the current volume, with $V$ being the volume of the sample at zero (gauge) pressure.

Hildebrandt [22] studied a similar ODE to Eq. (59) for describing the pressure/volume response of lung. His model was expressed

ๆ. The internal alveolar pressure $P$ is the negative of the external hydrostatic pressure $p=-\frac{1}{3} \operatorname{tr}(\boldsymbol{\sigma})=$ $-\frac{1}{3}|\mathbf{F}|^{-1} \operatorname{tr}(\boldsymbol{s})$, where $|\mathbf{F}|=v / V$. 
in terms of lung compliance, i.e., $\mathrm{d} V / \mathrm{d} P$; whereas, our model is described in terms of lung stiffness, viz., $\mathrm{d} P / \mathrm{d} V$. The right-hand sides of both of our differential equations are functions of pressure $P$. In his model, pressure is the independent variable; whereas, in our model, pressure is the dependent variable.

\subsection{Simple Shear}

There is not much simple about simple shear in finite deformations.

For the material model considered herein, to ensure that $P_{33}=0$, i.e., that the plane-stress assumption holds, it follows that the transverse stretch $\lambda_{w}$ must evolve according to the ODE

$$
\frac{\mathrm{d} \lambda_{w}}{\lambda_{w}}=-\frac{\alpha+2 \beta-2}{2(\lambda+2 \mu)} \mathrm{s}_{\tau} \mathrm{d} \gamma,
$$

which will be zero valued only when $\alpha=0$ and $\beta=1$, i.e., whenever the material is a grade-zero hypo-elastic solid [53].

Given the above constraint equation, the shear stress is described through an effective shear modulus where

$$
\begin{aligned}
\tilde{\mu} & =\frac{\mathrm{d} \mathrm{s}_{\tau}}{\mathrm{d} \gamma} \\
& =\mu-\frac{1}{2}\left(\mathrm{~s}_{1}-\mathrm{s}_{2}\right)+\frac{1}{2} \beta\left(\mathrm{s}_{1}+\mathrm{s}_{2}\right) \\
& \quad-\frac{(\alpha+2 \beta-2)^{2}}{4(\lambda+2 \mu)} \mathrm{s}_{\tau}^{2},
\end{aligned}
$$

where there is a linear dependence upon the normal stresses $s_{1}$ and $s_{2}$, and a quadratic dependence upon the shear stress $s_{\tau}$.

The normal stress acting in the direction of shearing is governed by

$$
\begin{aligned}
\frac{d s_{1}}{d \gamma} & =2 s_{\tau} \\
& +\frac{\left(4 \mu-\alpha s_{1}\right)(\alpha+2 \beta-2)}{4(\lambda+2 \mu)} s_{\tau},
\end{aligned}
$$

while the normal stress acting orthogonal to the direction of shearing is governed by

$$
\frac{d \mathrm{~s}_{2}}{\mathrm{~d} \gamma}=\frac{\left(4 \mu-\alpha \mathrm{s}_{2}\right)(\alpha+2 \beta-2)}{4(\lambda+2 \mu)} \mathrm{s}_{\tau},
$$

so simple shear is described by a non-linear system of coupled ODEs.

\subsection{Inflation/Compression}

In this experiment a lobe of lung is inflated to a volume of $v=\lambda_{v}^{3} V$ at an alveolar pressure of $P$ that associates with a KIRCHHOFF stress of $\mathrm{s}_{p}$, as established in Eq. (60). From this preset (or residual) state of stress, an axial compressive perturbation of $s_{\Delta}$ is imposed to which the lung responds with a relative stretching of $\lambda_{\ell}^{\Delta}$ and $\lambda_{w}^{\Delta}$ in the applied and transverse loading directions, respectively.

From the equations governing this BVP for our material model (as put forward in §5.5), one observes from the 11-component that

$$
\begin{aligned}
-\mathrm{ds}_{\Delta}=(\lambda & \left.+2 \mu+(\alpha+2 \beta)\left(\mathrm{s}_{p}-\mathrm{s}_{\Delta}\right)\right) \frac{\mathrm{d} \lambda_{\ell}^{\Delta}}{\lambda_{\ell}^{\Delta}} \\
& +\left(2 \lambda+\alpha \frac{1}{2}\left(4 \mathrm{~s}_{p}-\mathrm{s}_{\Delta}\right)\right) \frac{\mathrm{d} \lambda_{w}^{\Delta}}{\lambda_{w}^{\Delta}}
\end{aligned}
$$

while from the 22-component one gets

$$
\begin{aligned}
& \mathrm{ds}_{\Delta}=\left(2 \lambda+\alpha \frac{1}{2}\left(4 \mathrm{~s}_{p}-\mathrm{s}_{\Delta}\right)\right) \frac{\mathrm{d} \lambda_{\ell}^{\Delta}}{\lambda_{\ell}^{\Delta}} \\
& +\left(4(\lambda+\mu)+2(\alpha+\beta)\left(2 \mathrm{~s}_{p}+\mathrm{s}_{\Delta}\right)\right) \frac{\mathrm{d} \lambda_{w}^{\Delta}}{\lambda_{w}^{\Delta}},
\end{aligned}
$$

such that when added one arrives at an expression for an effective Poisson's ratio of

$$
\begin{aligned}
& \frac{\mathrm{d} \lambda_{w}^{\Delta} / \lambda_{w}^{\Delta}}{\mathrm{d} \lambda_{\ell}^{\Delta} / \lambda_{\ell}^{\Delta}}=-\frac{1}{2} \\
& \times \frac{3 \lambda+2 \mu+3 \alpha\left(\mathrm{s}_{p}-\mathrm{s}_{\Delta} / 2\right)+2 \beta\left(\mathrm{s}_{p}-\mathrm{s}_{\Delta}\right)}{3 \lambda+2 \mu+3 \alpha\left(\mathrm{s}_{p}+\mathrm{s}_{\Delta} / 4\right)+2 \beta\left(\mathrm{s}_{p}+\mathrm{s}_{\Delta} / 2\right)},
\end{aligned}
$$


that in the limit of $s_{\Delta} \rightarrow 0$ (which implies that $\lambda_{\ell}^{\Delta} \rightarrow 1$ and $\lambda_{w}^{\Delta} \rightarrow 1$ ) becomes

$$
\frac{\mathrm{d} \lambda{ }_{w}^{\Delta}}{\mathrm{d} \lambda_{\ell}^{\Delta}}=-\frac{1}{2}
$$

independent of the value of any material parameter in the model. In other words, compressive perturbations are isochoric in neighborhoods around a state of uniform inflation.

Taking the result from Eq. (67) and substituting it into either expression for $\mathrm{ds}_{\Delta}$, while taking the limits $\mathrm{s}_{\Delta} \rightarrow 0$ and $\lambda_{\ell}^{\Delta} \rightarrow 1$, one arrives at a modulus of response of

$$
M \doteq-\frac{\mathrm{ds}_{\Delta}}{\mathrm{d} \lambda_{\ell}^{\Delta}}=2\left(\mu+\beta \mathrm{s}_{p}\right),
$$

where the contributions that arise from parameters $\lambda$ and $\alpha$ cancel out. This implies that the stiffness of the lung increases with pressure, which is experimentally observed [30].

\section{Experiments}

It is well known that there is substantial hysteresis in the pressure/volume curves of inflating/ deflating lungs [26]. This is partially caused by the viscoelastic attributes of the tissue [48]; but predominantly caused by the surfactant cycle that aids in the mechanics of breathing [40, 43]. Neither of these effects are to be addressed in this preliminary study. Here we only interest ourselves with the elastic contributions to the mechanical response of lung. Time-dependent effects are to be the primary research emphasis during the second year of our funding.

The experimental data of LAI-Fook et al. [30] are used to demonstrate applicability of our hypo-elastic material model in describing the mechanical response of deflating, spongy, lung tissue (i.e., parenchyma). The data used here came from an excised, lower, left lobe of a dog's lung. The pressure/volume data acquired from this experiment are listed in Table 2.

\begin{tabular}{|c|c|c|c|}
\hline$v$ & $\lambda_{v}^{3}=v / V$ & $P$ & $\mathrm{~s}_{p}=P \lambda_{v}^{3}$ \\
\hline 240 & 1 & 0 & 0 \\
450 & 1.90 & 4 & 7.6 \\
590 & 2.45 & 7 & 17.2 \\
660 & 2.75 & 9 & 24.8 \\
730 & 3.05 & 12 & 36.6 \\
\hline
\end{tabular}

Table 2: Pressure/volume data from the lower left lobe of a dog's lung. The reported volumes are in $\mathrm{ml}$, and the pressures are in $\mathrm{cm} \mathrm{H}_{2} \mathrm{O}$. The data were extracted from Fig. 1 in LAI-FooK et al. [30].

\begin{tabular}{|l||c|c||c|c|}
\hline Species & $\lambda\left(\mathrm{cm} \mathrm{H}_{2} \mathrm{O}\right)$ & $\alpha$ & $\mu\left(\mathrm{cm} \mathrm{H}_{2} \mathrm{O}\right)$ & $\beta$ \\
\hline Dog & 1.8 & 3.6 & 0.2 & 0.38 \\
\hline
\end{tabular}

Table 3: Hypo-elastic material parameters for lung parenchyma.

The resulting model parameters that pertain to this data set are tabulated in Table 3. It is curious to point out that $\alpha$ is the hypo-elastic analog to $\lambda$, while $\beta$ is its analog for $\mu$. Furthermore, these hypo-elastic Fung parameters are about twice the size of their LAMÉ counterparts, while $\lambda$ and $\alpha$ are about an order in magnitude greater than $\mu$ and $\beta$. There is therefore a certain consistency amongst these parameters.

Deflation data are used in this investigation, as they are the data that are the closest to being quasi-static, i.e., their non-elastic contributions are thought to be minimal. In the experiments of LAI-Fook et al. [30], each lobe of lung was prepared by degassing it in a vacuum jar. The lobes were then pressurized to total lung capacity (TLC), about 25-30 $\mathrm{cm} \mathrm{H}_{2} \mathrm{O}$, and allowed to equilibrate there. (At TLC there is about 12-14 milliliters of gas per gram of tissue, while at atmospheric pressure, i.e., a gauge pressure of $0 \mathrm{~cm} \mathrm{H}_{2} \mathrm{O}$, there is about 1-2 milliliters of trapped gas in the alveoli per gram of tissue [26].) After equilibrating at TLC, the lobes were statically decompressed to a sequence of 


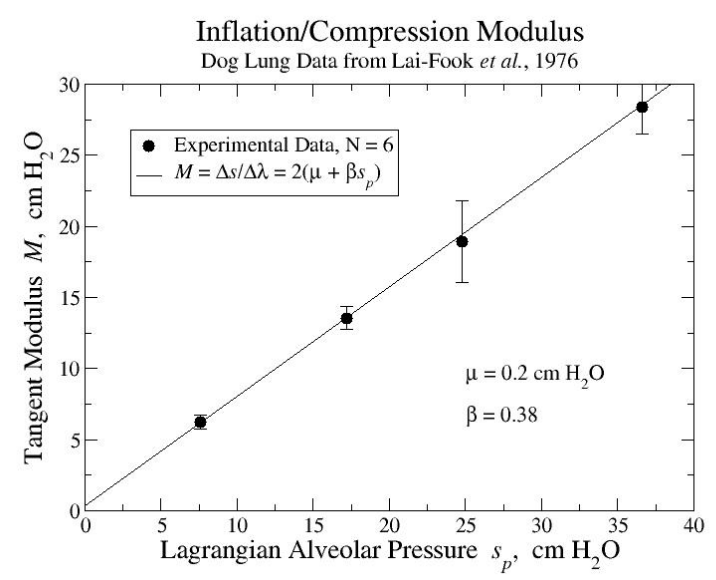

Figure 4: A least-squares fit of Eq. (68) to the experimental data of LAI-Fook et al. [30, Fig. 3], with an $R^{2}$ error of 0.9995 . Error bars are \pm 1 standard deviation.

targeted alveolar pressures, where perturbations in the loading were applied, e.g., compressions were superimposed onto an otherwise hydrostatic state of stress, while holding the alveolar pressure constant. This is the experiment whose BVP is described in $\$ 5.5$, and for which Eq. (68) determines its effective modulus.

A fit of Eq. (68) to the experimental data of LAI-FooK et al. [30, Fig. 3] is presented in Fig. 4. The hypo-elastic Fung parameter $\beta$ describes a stiffening of lung with increasing alveolar pressure $\mathrm{s}_{p}$, which is seen to dominate the classic elastic parameter, viz., that attributed to the shear modulus $\mu$. From this experiment, one can ascertain two of the four material parameters in our hypo-elastic model for parenchyma: $\mu$ establishes the intercept, while $\beta$ describes the slope.

As a point of curiosity, we converted the stress type from KirchHoff stress $s$ to CAUCHY stress $\sigma$ via $\sigma_{\Delta}=\mathrm{s}_{\Delta} /|\mathbf{F}|=\mathrm{s}_{\Delta} V / v$, thereby changing the way by which stress is computed along both axes in Fig. 4, as specified in

$$
M \cong-\frac{\mathrm{d} \sigma_{\Delta}}{\mathrm{d} \lambda_{\ell}^{\Delta}}=2(\mu+\beta P),
$$

where now the alveolar pressure $P$ is the stress

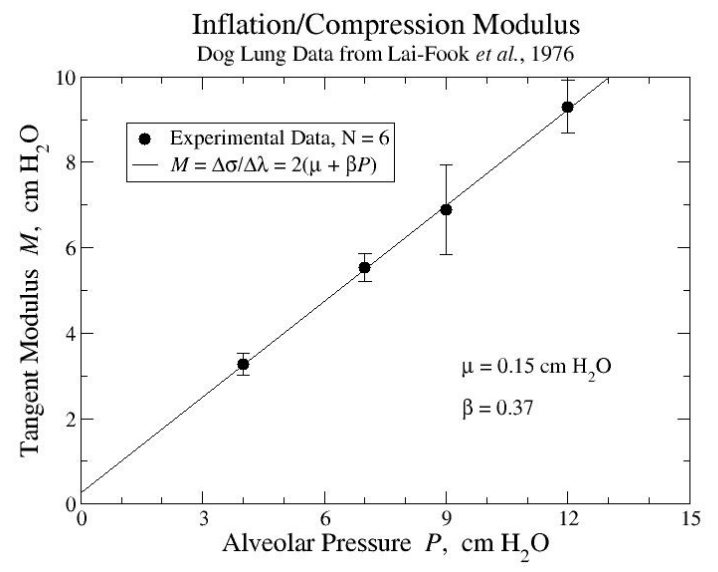

Figure 5: A least-squares fit of Eq. (69) to the experimental data of LAI-Fook et al. [30, Fig. 3], with an $R^{2}$ error of 0.9990. Error bars are \pm 1 standard deviation.

variable, which is the pressure controlled in an experiment. This was done to see if differences would arise from a least squares analysis. The outcome is presented in Fig. 5. For all practical purposes, no significant differences were observed, because the shear modulus is so small. This means that the experimentalist can choose to use either Eulerian (69) or Lagrangian (68) measures of stress to acquire these parameters, so long as modulus $M$ is constructed correctly.

For the BVP put forward in $\S 5.5$, our hypoelastic model for parenchyma predicts that uniaxial excursions from a state of uniform inflation in lung will be isochoric, i.e., the effective PoIsson's ratio will be $1 / 2$, as derived in Eq. (67), independent of the state of pressure $P$, and independent of the values for the model's parameters. So, although one cannot extract useful parametric values from such measurements, one can test the validity of the theory. This is done in Fig. 6 using the same experimental data set that was used to quantify $\mu$ and $\beta$ above. What one sees is that the measured values for Poisson's ratio are indeed independent of alveolar pressure $P$, and their values are slightly less than, but very close to the predicted value of $1 / 2$. (The classical theory of elasticity bounds 


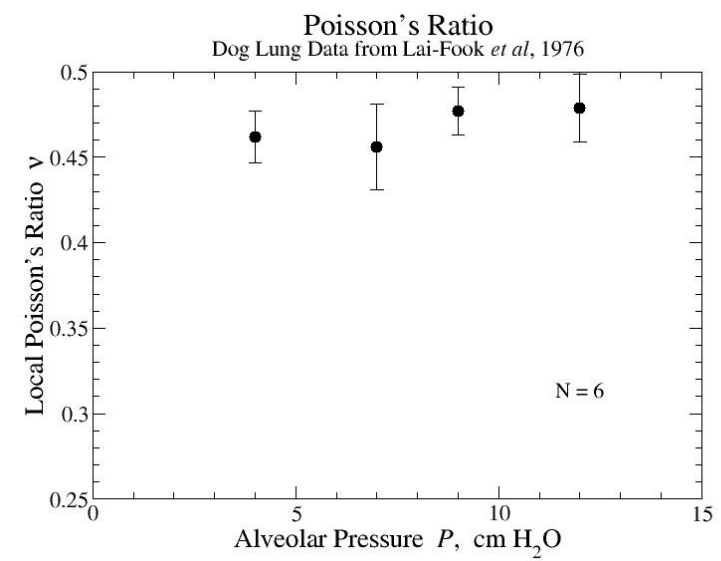

Figure 6: PoIsson's ratio during compression excursions from states of uniform hydrostatic pressure. Experimental data are from LaI-Fook et al. [30, Fig. 3]. Error bars are \pm 1 standard deviation.

Poisson's ratio from above at $1 / 2$.) These data provide yet another bit of evidence confirming our choice of selecting a hypo-elastic material model for representing the elastic behavior of lung tissue.

The final experiment called upon to complete our model's parameterization is that of a deflating pressure/volume curve. Here we use the pressure/volume curve of LAI-Fook et al. [30, Fig. 1], from which compression excursions were taken that produced the experimental data that are found in Figs. 4-6; in other words, all data used for parameter estimation in this study came from a single specimen. This allows us to assess the viability of our model without having to address specimen-to-specimen variability, which is large when sampling from a biological population. The model's fit to these data is shown as the solid curve in Fig. 7.

\section{$8 \quad$ Structure of Lung}

SEM photos of rat lung are provided in Figs. 8-11 with increasing magnifications."

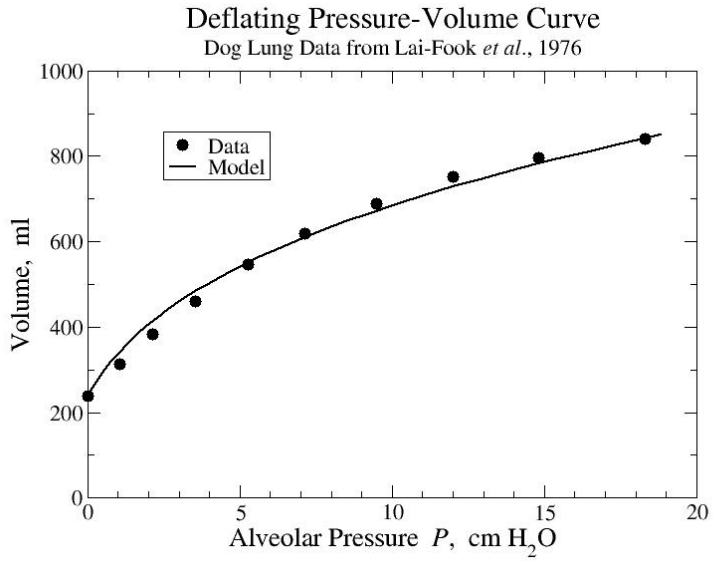

Figure 7: The quasi-static pressure/volume response of deflating lung, and the ability of our hypo-elastic material model to represent it, with an $R^{2}$ error of 0.994. Experimental data are from LAI-Fook et al. [30, Fig. 1].

In Fig. 8, the parenchyma is seen to be a fairly uniform sea of tiny alveolar sacks that are periodically perforated by alveolar ducts for transporting the air in and out of the lung. This photo focuses in on a roughly $1 \mathrm{~mm} \times 1 \mathrm{~mm}$ sectioning of lung.

In Fig. 9, zooming in on this alveolar landscape, while focusing our attention on the forth quadrant in the photo, we observe that the alveolar mouths actually comprise a large area fraction of the walls of the alveolar ducts [57]. These airways are highly perforated.

Continuing to zoom in, in the central region of Fig. 10 we see the mouths of several alveolar sacs. These annuli have pentagonal shapes, and are comprised of heavier cords of fiber than

$\|$. Protocol for SEM sample preparation for rat lung tissue: Primary fixation consisted of $2.5 \%$ glutaraldehyde in a $0.1 \mathrm{M}$ phosphate buffer and $1.0 \%$ sucrose $+\mathrm{CaCl}_{2}, \mathrm{pH} 7.2$, at $4^{\circ} \mathrm{C}$ for $2 \mathrm{hr}$. Post fixation was with $1.0 \% \mathrm{OsO}_{4}$ in buffer for $1 \mathrm{hr}$. at $4^{\circ} \mathrm{C}$. Both fixation steps were followed by 4 buffer washes at $15 \mathrm{~min}$. each. Dehydration was in a graded series of acetone. Samples were critical point dried (Denton DCP-1), sputter coated with gold (Denton Desk II) twice at $40 \mathrm{mAmp}$ and 50 Torr for $2 \mathrm{~min}$., and then viewed with the JEOL 5400 SEM at $15 \mathrm{kV}$. 


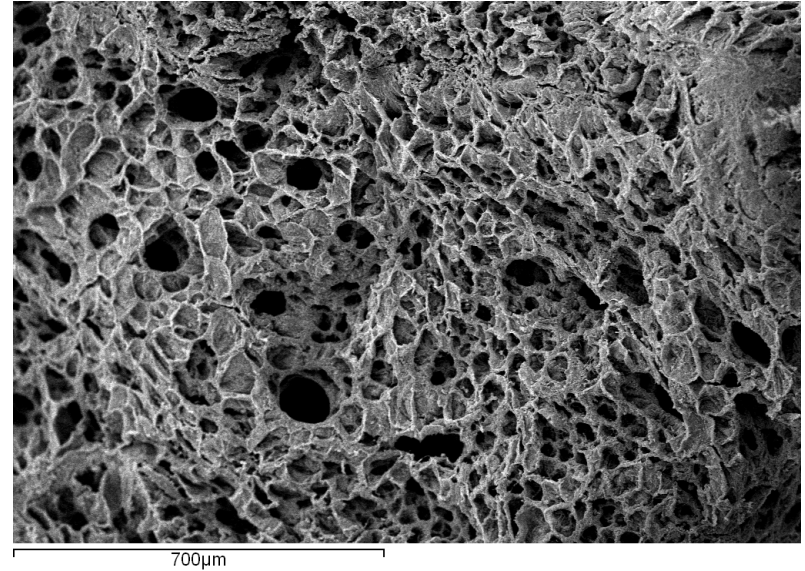

Figure 8: SEM photograph of rat lung.

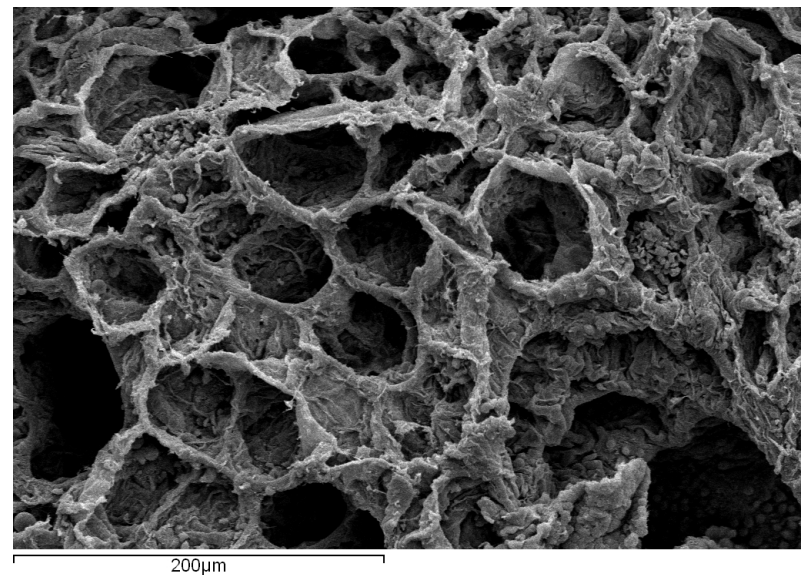

Figure 9: SEM photograph of rat lung.

are found elsewhere throughout their structures [35]. The pentagonal facets of alveolar walls are shared membranes between two neighboring alveoli. Random fiber filaments (elastin and collagen) are seen to thread from the septa, crisscrossing their faces [45].

The final photo in Fig. 11, taken at the same magnification of Fig. 10, does a nice job of showing the capillary network that intertwines with the fibrous structure of the alveolar walls [26]. This is especially visible in the second quadrant of the photo. It is here that gases exchange between air and blood via diffusion across the capillary walls $[57,58]$.

It is photographs like these that provide the impetus for the modeling efforts that follow.

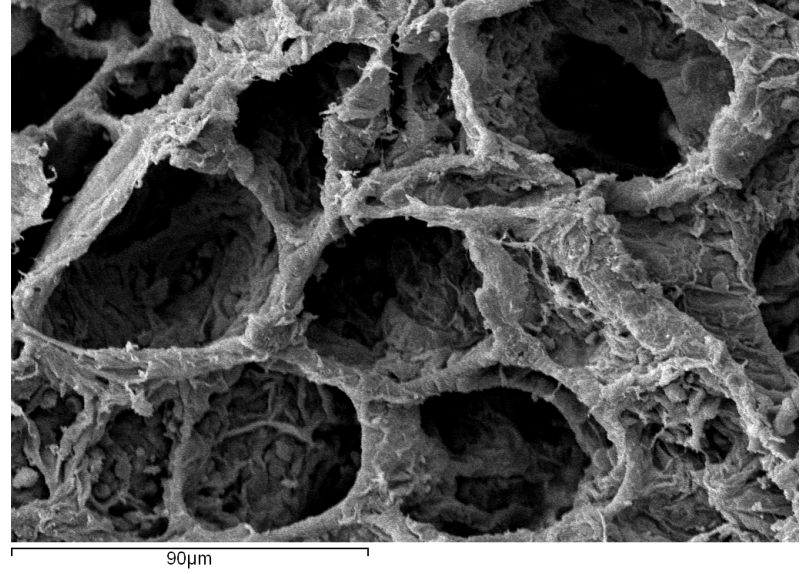

Figure 10: SEM photograph of rat lung.

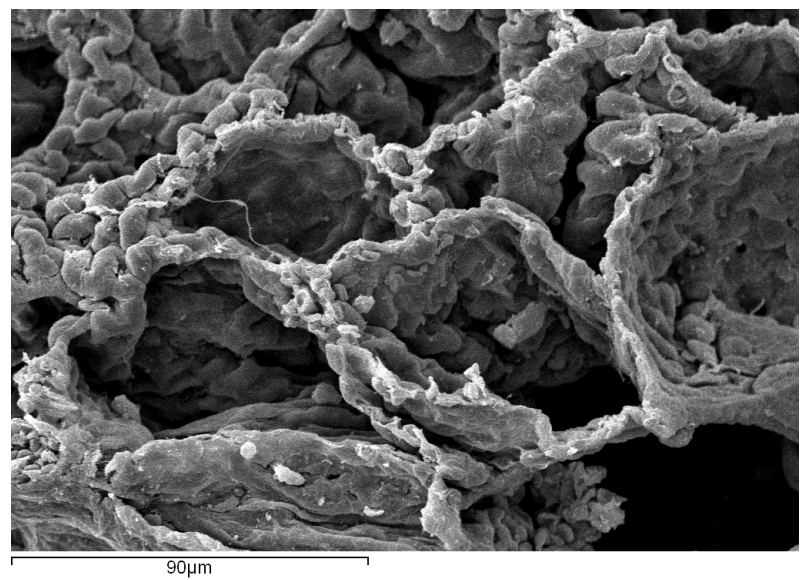

Figure 11: SEM photograph of rat lung.

\section{Dodecahedral Model}

"Constitutive equations are phenomenological. They are regarded as empirical by experimenters, and axiomatic by mathematicians. In biomechanics, we often try to derive them on the basis of microstructure $\ldots$ in order to gain a better understanding, or to get some guidance to the mathematical form."

FUNG [19, pg. 431]

Typical alveoli are fourteen sided polyhedra with one face being open as its mouth to an alveolar duct [26]. To capture the microstructure of lung, researchers have modeled 
both the alveolus and alveolar ducts. Two different geometric shapes are usually employed when modeling an alveolus: a dodecahedron, as introduced by Frankus \& LeE [9], and a truncated octahedron, as introduced by DALE, Matthews \& Schroter [5]. A dodecahedron is a twelve sided polyhedron with each face being a regular pentagon. A truncated octahedron is a pair of regular pyramids stacked bottom to bottom, which is an octahedron, with its six points lopped off. This produces a fourteen sided polyhedron with six faces that are squares and eight faces that are hexagons, where like shapes have like dimensions.

The truncated octahedron is volume filling, and is therefore the preferred geometry to use when constructing assemblages of these alveoli for building microstructural models to be numerically solved through finite element methods for the purpose of homogenizing the response up to the macroscopic level, i.e., at a continuum mass point, e.g., $[5,6,7]$.

The dodecahedron is isotropic, and is therefore the preferred geometry to use when a single alveolus is to be homogenized in closed form up to the macroscopic level, e.g., [4, 29]. Here the isotropy of the microstructure ensures an isotropic macro response. We re-emphasize that parenchyma is isotropic; whereas, lung is described by a heterogeneous boundary-value problem.

Because we are seeking a model for parenchyma to use as a material model within OpenFOAM $($, the analytical approach of KIMMEL and BUDIANSKY is preferred over the numerical approach of DaLe, DenNy and Schroter for describing the continuum response of parenchyma at the GAuss points in a CFD model of lung.

\subsection{Fiber Constitutive Relations}

With only a few exceptions, e.g., in [27] where the alveolar faces are modeled as membranes, all of these geometric models for the alveolar microstructure of lung are constructed as pinned space trusses, whose bar elements are modeled as collagen and elastin fibers that only transmit tensile forces. There are, however, differences between the force/extension laws used, i.e., the assumed constitutive responses assigned to describe the behavior of the links that make up these space trusses.

In the models of Kimmel et al. [4, 28, 29], the tangent modulus for the fiber response is assumed to obey

$$
\frac{\dot{f}}{f}=B \frac{\dot{L}}{L},
$$

which integrates to

$$
f=\left(\frac{L}{L_{0}}\right)^{B}-1,
$$

where $f$ is the force of traction carried by a typical fiber, or truss element, while $L$ is its length along a septal junction. $L$ associates with an edge along one of the pentagon faces, with $L_{0}$ denoting its reference length at the end of total exhale, i.e., at zero alveolar pressure. This model has a single material parameter, viz., $B$, that relates the logarithmic rates of traction and length. Examining Eq. (71), we see that $f$ is actually a measure of deformation or strain, not of force. What it lacks is a modulus.

In the paper of Denny \& Schroter [7], they settled in on a conglomerate constitutive description that includes models for both elastin and collagen fibers that are loaded in parallel. They consider elastin fibers to be Hookean with a stress/strain response of

$$
\sigma_{e}=c_{3} \epsilon,
$$

while collagen fibers are assumed to behave as

$$
\sigma_{c}=-c_{1} \ln \left(1-\frac{1}{c_{2}}\left(\mathrm{e}^{\epsilon}-1\right)\right),
$$


where $\sigma_{e}$ and $\sigma_{c}$ are the stresses in the elastin and collagen fibers, respectively, so $\sigma=\sigma_{e}+$ $\sigma_{c}$, with $\epsilon$ being the strain, and $c_{1}, c_{2}$, and $c_{3}$ denoting material constants. Exactly what measures of stress and strain they employ is not specified. If they are as defined by DALE et al. [5], which is quite likely, then these are measures of true stress and true strain. The tangent moduli associated with these models are [5]

$$
\begin{aligned}
& \mathrm{d} \sigma_{e} / \mathrm{d} \epsilon=c_{3}, \\
& \mathrm{~d} \sigma_{c} / \mathrm{d} \epsilon=\frac{c_{1} \mathrm{e}^{\epsilon}}{\left(1+c_{2}\right)-\mathrm{e}^{\epsilon}},
\end{aligned}
$$

neither of which captures the simplicity of FunG's law (24).

The constitutive models used by the research groups of Kimmel and Schroter have vastly different physical interpretations, and are substantively different from the material models that we propose to adopt below.

\subsection{Model Assumptions}

Applications of the principal of virtual work, following procedures put forward by BUDIANSKY \& Kimmel [4, 28], impose virtual displacements on the dodecahedron through which equivalent hypo-elastic moduli corresponding to the stimulated continuum are to be hopefully revealed.

A microscopic modeling of parenchyma is proposed that builds upon the following list of assumptions put forward by Kimmel and his colleagues, cf. [4, 29]:

- A regular, dodecahedral, space truss represents the geometry of an alveolus, in an averaged sense.

- All truss elements are pinned (carry no moments) and remain in tension.

- The alveolar mouth with its thicker fibers and open face is modeled like any of the other eleven pentagonal elements in the dodecahedron, i.e., the presence of a fictitious face is assumed to offset the differences in the observed fiber diameters.

- The geometry remains self-similar when subjected to an isotropic stress.

- The vector displacement at the center of each pentagonal face is the mean of the displacements at its five corners.

- On each pentagonal face, the displacement varies linearly within each of the five constituent triangles, which share a common corner at the center of the pentagon.

The area of a regular pentagon is

$$
A=1.72 L^{2},
$$

whose nominal diameter is

$$
D=1.48 L,
$$

when compared to a circle. Here $L$ is the length of a side of the pentagon.

A dodecahedron has a volume of [29]

$$
\bar{V}=7.76 L^{3},
$$

a nominal cross-sectional area (compared to a sphere) of

$$
\bar{A}=4.74 L^{2},
$$

and a nominal diameter (compared to a sphere) of

$$
\bar{D}=2.46 L,
$$

so all geometric values are expressed in terms of the septal length of a side of a pentagon within a dodecahedron. As a matter of notation, dodecahedral dimensions have a bar over them; whereas, pentagonal dimensions do not.

To dimension the alveoli of human lung, Sobin, Fung \& Tremer [45] measured the mean 
chord lengths, viz., $\bar{D}$, across individual alveoli sectioned from lungs that were fixed at different pressures. Samples were taken from 9 lungs distributed across the ages of 16 to $89 .{ }^{* *}$ At a transpulmonary pressure of $4 \mathrm{~cm} \mathrm{H}_{2} \mathrm{O}$, the measured mean chord length from 1423 samples was $\bar{D}=191 \pm 86 \mu \mathrm{m}$; at a pressure of $10 \mathrm{~cm} \mathrm{H}_{2} \mathrm{O}, \bar{D}=202 \pm 88 \mu \mathrm{m}$ from 1296 samples; and at a pressure of $14 \mathrm{~cm} \mathrm{H}_{2} \mathrm{O}$, $\bar{D}=235 \pm 99 \mu \mathrm{m}$ from 1083 samples. All reported errors pertain to plus/minus one standard deviation.

Where our approach will predominantly differ from those that exist in the literature resides in the following six assumptions:

- Each truss element assumes the behavior of incompressible cylinder of material described by its associated material type, while each face is modeled as an incompressible membrane.

- Elastin is modeled as a hyper-elastic neo-Hookean solid [1].

- Collagen is modeled as a hypo-elastic FunG-like solid [11].

- The elastin and collagen fiber networks act independently [35, 45]. Their elements are loaded in parallel.

- The concentration of surfactant $\Gamma$ is governed by three formulæ: one describing its liquid expansion, one its liquid condensation, and another its solid regime [40].

- The surface tension $\gamma$ caused by surfactant has the response of a uniformly stretched, neo-Hookean, elastic membrane whose elastic modulus varies with concentration $\Gamma$.

The first five of these assumptions are quite sound. The experimental data that support the last conjecture are marginal, if existent at all, e.g., the data of ScHüch et al. [43].

\subsubsection{Our First Assumption}

Although lung is a highly compressible material, the constituents that provide its structural integrity are, to a good approximation, incompressible. What this really means is that the ratio of their shear to bulk moduli is smaller than about one part in a hundred, which is common amongst soft solids. This being the case, all structural members of the dodecahedron are built with incompressible materials whose governing equations depend upon the material each member is made from, and the BVP imposed upon it.

\subsubsection{Our Second Assumption}

Elastin is a protein that is an isotropic polypeptide elastomer built from monomeric units that have between three and six peptides in each repeating unit with a total of about 100 monomers in a typical elastomer chain [55]. Elastin is also entropic above about $25^{\circ} \mathrm{C}$. Therefore, its physiochemical properties are compatible with those used to derive the classical theory of rubber elasticity from statistical mechanics [50].

The load bearing alveolar fibers are located predominantly along the septal junctions, i.e., along the pentagonal edges of the dodecahedron. As these are taken to be pinned truss elements loaded under tension only, it follows that the elastin fibers in this microstructural model are described by uniaxially stretched neo-Hookean rods so that $T_{e}=\mu_{e}\left(\lambda-\lambda^{-2}\right)$ [50] or equivalently

$$
f_{e}=\mu_{e} A_{0}^{e}\left(\frac{L}{L_{0}}-\frac{L_{0}^{2}}{L^{2}}\right),
$$

and whose structural tangent modulus is

$$
\frac{\mathrm{d} f_{e}}{\mathrm{~d} L / L}=3 \mu_{e} A_{0}^{e} \frac{L_{0}^{2}}{L^{2}}+f_{e},
$$

**. SobIN et al. [45] documented an age effect in these data that has been averaged over here, i.e., ignored. 
wherein $T_{e}=f_{e} / A_{0}^{e}$ is the stress of traction, $f_{e}$ is the force of traction, and $A_{0}^{e}$ is the initial cross-sectional area of a typical elastin fiber, with $\lambda=L / L_{0}$ being the stretch of a truss member whose initial $L_{0}$ and current $L$ lengths associate with the dimension of a pentagonal edge, while $\mu_{e}$ is the shear modulus of elastin whose value is $280 \mathrm{kPa}$ at $37^{\circ} \mathrm{C}$ [55].

Matsuda, Fung \& Sobin [35] measured the diameter of elastin fiber bundles at the alveolar mouths in human lung at $7.11 \pm 2.93 \mu \mathrm{m}$, with the reported error being plus/minus one standard deviation from a sample size of 450 taken from a single lung. Sobin, Fung \& Tremer [45] measured the elastin fiber diameter at $d_{e}=$ $1.106 \pm 0.552 \mu \mathrm{m}$ in the inter-alveolar septa from 2030 samples taken from 5 lungs, with $A_{0}^{e}=\pi d_{e}^{2} / 4$.

\subsubsection{Our Third Assumption}

Much of the author's research efforts over the past five years has focused around the observation that the elastic behavior of collagenous materials is well described by a hypo-elastic representation of Fung's law [11, 14]. Collagen, like elastin, contributes to the transmission of tractions along the truss elements in the dodecahedron, and as such, is modeled via stretchable uniaxial rods whose material response is described by $\lambda^{2} \mathrm{~d} T_{c} / \mathrm{d} \lambda=3 \mu_{c}+\beta \lambda T_{c}$ [11] that when integrated becomes

$$
f_{c}=\frac{3 \mu_{c} A_{0}^{c}}{\beta+1}\left(\frac{L^{\beta}}{L_{0}^{\beta}}-\frac{L_{0}}{L}\right),
$$

and whose structural tangent modulus is

$$
\frac{\mathrm{d} f_{c}}{\mathrm{~d} L / L}=3 \mu_{c} A_{0}^{c} \frac{L_{0}}{L}+\beta f_{c},
$$

wherein $T_{c}=f_{c} / A_{0}^{c}$ is the stress of traction, $f_{c}$ is the force of traction, and $A_{0}^{c}$ is the initial cross-sectional area of a typical collagen fiber, while $\mu_{c}$ is the small-strain shear modulus of collagen, and $\beta$ is FunG's parameter in Eq. (24).
Matsuda, Fung \& Sobin [35] measured the diameter of collagen fiber bundles at alveolar mouths in human lung at $4.98 \pm 2.24 \mu \mathrm{m}$, with the reported error being plus/minus one standard deviation from a sample size of 3,095 taken from a single lung. Sobin, Fung \& Tremer [45] measured the collagen fiber diameter at $d_{c}=1.053 \pm 0.539 \mu \mathrm{m}$ in the inter-alveolar septa from 6,902 samples taken from 14 lungs, with $A_{0}^{c}=\pi d_{c}^{2} / 4$.

\subsubsection{Our Forth Assumption}

The extensive histological studies of MatsudA et al. [35] and of Sobin et al. [45] provide no evidence suggesting that collagen and elastin fibers are either mechanically or chemically interconnected in some way, although that was not an issue specifically addressed in their papers.

\subsubsection{Our Fifth Assumption}

OTIs et al. [40] decomposed the kinetics governing surfactant concentration $\Gamma$ into three regimes.

The first region is the liquid-expansion regime, where $\Gamma<\Gamma^{\star}$ with $\Gamma^{\star}$ being the maximum equilibrium concentration of surfactant at the air/liquid interface, i.e., within the surface experiencing tension. Here they assume that LANGMUIR kinetics control the transport of surfactant between the interface and the fluid it contains, as described by

$$
\begin{aligned}
\frac{\mathrm{d}(\Gamma A)}{\mathrm{d} t} & =A\left(k_{1} C\left(\Gamma^{\star}-\Gamma\right)-k_{2} \Gamma\right), \\
& =c_{1} \Gamma^{\star} A-c_{2} \Gamma A,
\end{aligned}
$$

where $k_{1}$ and $k_{2}$ are the LANGMUIR absorption and desorption coefficients, with $c_{1}=k_{1} C$ and $c_{2}=k_{1} C+k_{2}$.

The second region is the liquid-condensed regime, where $\Gamma^{\star} \leq \Gamma \leq \Gamma_{\max }$. Here Oтіs 
et al. consider that the concentration of surfactant can only change if the area changes, so in this regime

$$
\Gamma=\Gamma^{\star} \frac{A^{\star}}{A},
$$

up to a maximum value of $\Gamma=\Gamma_{\max }$.

The third and final region is the solid regime, where the concentration of surfactant within the air/liquid interface is saturated at

$$
\Gamma=\Gamma_{\max }
$$

Here the surface tension $\gamma$ is at its minimum.

Values reported in the literature for these constants are [8]: $c_{1}=1.168 \mathrm{~s}^{-1}$ and $c_{2}=$ $1.184 \mathrm{~s}^{-1}$, with $\gamma\left(\Gamma_{\max }\right)=2 \mathrm{dyn} / \mathrm{cm}$ and $\gamma\left(\Gamma^{\star}\right)=22.2 \mathrm{dyn} / \mathrm{cm}$.

\subsubsection{Our Sixth Assumption}

We can strengthen our case for the fifth assumption by considering that surfactant is a viscoelastic fluid made up of a variety of macromolecular complexes being held in suspension. These complexes are comprised of $90 \%$ lipids, $10 \%$ proteins, and small amounts of carbohydrate. Dipalmitoylphosphatidylcholine (DPPC) makes up about half of this lipid content, and is primarily responsible for the surface tension reducing property of the surfactant [43].

These macromolecules are not so different in configuration or size from those for which the rubberlike liquid applies [32]. The rubberlike liquid is a viscoelastic material model derived from statistical physics [33] whose derivation parallels that of the neo-Hookean elastic solid [50], except now the cross-links between molecular chains are no longer permanent. It is because of surfactant's importance to lung mechanics due to its surface tension properties, not its bulk fluid properties, that we can consider the 'skin' of this fluid as being an elastic membrane, ignoring the effect of the bulk fluid residing underneath.
From this line of reasoning, it is supposed that a neo-Hookean membrane under equi-biaxial (or isotropic) traction describes the fluid surface so that $\gamma=\mu_{s}(\Gamma)\left(\lambda-\lambda^{-5}\right)$ [50] or

$$
\gamma=\mu_{s}(\Gamma)\left(\frac{L}{L_{0}}-\frac{L_{0}^{5}}{L^{5}}\right),
$$

where $\gamma=f_{s} / D$ is the surface traction, $f_{s}$ is the force of traction, and $D$ is the length of traction, which is taken to be the nominal diameter of a pentagon in the dodecahedron. Self-similarity allows one to replace $\lambda=D / D_{0}$ with $L / L_{0}$ and $\lambda^{2}=D^{2} / D_{0}^{2}$ with $A / A_{0}$, so the relevant tangent modulus is described by

$$
\frac{\mathrm{d} \gamma}{\mathrm{d} A / A}=3 \mu_{s}(\Gamma) \frac{L_{0}^{5}}{L^{5}}+\frac{1}{2} \gamma,
$$

where $\mu_{s}$ has units of force per unit length, not force per unit area as is the case for $\mu_{c}$ and $\mu_{e}$. This shear modulus varies with surfactant concentration, i.e., $\mu_{s}(\Gamma)$, which is a topic for future work where a link between assumptions 5 and 6 is to be built.

This completes our construction for the constitutive relations that are inherent in this microstructure.

\subsection{Bulk Modulus}

Following the method of analysis laid out by Budiansky \& Kimmel [4, 28], the principal of virtual work is applied to a dodecahedral space truss of volume $\bar{V}$ loaded to a state of hydrostatic (or alveolar) pressure $P$. This in turn produces identical forces of reaction $f=$ $f_{c}+f_{e}$ in its 30 members of length $L$, and surface tensions $\gamma$ across its 12 faces of area $A$ such that

$$
P \mathrm{~d} \bar{V}=30 f \mathrm{~d} L+12 \gamma \mathrm{d} A .
$$

Self-similar growth of a dodecahedron under increasing pressure $P$ requires that

$$
\frac{\mathrm{d} \bar{V}}{\bar{V}}=3 \frac{\mathrm{d} L}{L}=\frac{3}{2} \frac{\mathrm{d} A}{A},
$$


that when combined with Eq. (89) produces

$$
P \bar{V}=10 f L+8 \gamma A,
$$

which, from Eqs. (75 \& 77), predicts a state of hydrostatic pressure in an alveolus of

$$
P=1.29 \frac{f_{e}+f_{c}}{L^{2}}+1.77 \frac{\gamma}{L} .
$$

Substituting in the constitutive equations (80, $82 \& 87$ ) leads to

$$
\begin{aligned}
& P=1.29\left\{\frac{\mu_{e} A_{0}^{e}}{L_{0}^{2}}\left(\frac{L_{0}}{L}-\frac{L_{0}^{4}}{L^{4}}\right)\right. \\
&+\left.\frac{3 \mu_{c} A_{0}^{c}}{(\beta+1) L_{0}^{2}}\left(\frac{L^{\beta-2}}{L_{0}^{\beta-2}}-\frac{L_{0}^{3}}{L^{3}}\right)\right\} \\
&+1.77 \frac{\mu_{s}(\Gamma)}{L_{0}}\left(1-\frac{L_{0}^{6}}{L^{6}}\right),
\end{aligned}
$$

whose comparison to experimental data and the hypo-elastic model is left for future work.

Differentiating Eq. (91) gives

$$
\begin{aligned}
\bar{V} \mathrm{~d} P & +P \mathrm{~d} \bar{V} \\
= & 10(L \mathrm{~d} f+f \mathrm{~d} L) \\
& +8(A \mathrm{~d} \gamma+\gamma \mathrm{d} A),
\end{aligned}
$$

from which a local or tangent bulk modulus can be defined via

$$
K=\frac{\mathrm{d} P}{\mathrm{~d} \bar{V} / \bar{V}},
$$

so that upon combining Eqs. $(89,90 \& 94)$ with Eq. (95) one is lead to

$$
\begin{aligned}
K=10( & \left.\frac{\mathrm{d} f}{\mathrm{~d} L / L}-2 f\right) \frac{\mathrm{d} L}{\mathrm{~d} \bar{V}} \\
& +8\left(\frac{\mathrm{d} \gamma}{\mathrm{d} A / A}-\frac{1}{2} \gamma\right) \frac{\mathrm{d} A}{\mathrm{~d} \bar{V}},
\end{aligned}
$$

that after introducing Eqs. $(75,77 \& 90)$ it becomes

$$
\begin{aligned}
K=\frac{0.43}{L^{2}} & \left(\frac{\mathrm{d} f}{\mathrm{~d} L / L}-2 f\right) \\
& +\frac{1.18}{L}\left(\frac{\mathrm{d} \gamma}{\mathrm{d} A / A}-\frac{1}{2} \gamma\right) .
\end{aligned}
$$

At this point the constitutive equations (81, $83 \& 88$ ) for the various element types in the dodecahedron combine with the above formula leading to our final expression for the local bulk modulus

$$
\begin{array}{r}
K=0.43\left(3 \frac{\mu_{e} A_{0}^{e}}{L_{0}^{2}} \frac{L_{0}^{4}}{L^{4}}-\frac{f_{e}}{L^{2}}\right. \\
\left.+3 \frac{\mu_{c} A_{0}^{c}}{L_{0}^{2}} \frac{L_{0}^{3}}{L^{3}}+(\beta-2) \frac{f_{c}}{L^{2}}\right) \\
+3.54 \frac{\mu_{s}(\Gamma)}{L_{0}} \frac{L_{0}^{6}}{L^{6}}
\end{array}
$$

This completes our studies for the first year of this contractual effort.

\section{Summary}

In this report we have demonstrated that hypo-elasticity provides a viable alternative to hyper-elasticity for the modeling of lung parenchyma. Hypo-elastic models are natural candidates for use in CFD codes, which motivated this study. In these codes, velocity and it its gradient are the independent kinematic variables, as they are in hypo-elasticity.

A useful feature of the proposed hypo-elastic material model is that its characterization can be accomplished from a single experimental protocol: Inflate a lobe of lung to its TLC; quasi-statically deflate it to a set of targeted alveolar pressures; apply uniaxial compressive perturbations while holding the pressure fixed in the lung. From these pressure, volume, and compressive load/ deflection perturbations and measurements, one has sufficient experimental data to apply Eqs. (61 \& 69) for the purpose of estimating the model's parameters.

It is conjectured that, and preliminary steps have been taken toward proving that the material parameters for this hypo-elastic model will associate with the response of a 
REFERENCES

micro-mechanics based model. Such a capability would permit the affects of disease states to be modeled in a fashion that is not currently possible.

\section{Acknowledgments}

Professor SAlly Shepardson from the Biology Department at Saginaw Valley State University is thanked for preparing the samples and then taking the SEM photos of rat lung that appear in this report.

OPENFOAM $\cap$ is a registered trade mark of OpenCFD Limited, the producer of the OpenFOAM@software.

Data taken from published sources were re-digitized by scanning an image of the required figure, and then digitizing the individual datum points from the scanned image using the g3data software package of JonAs FRANTz. http://www . frantz.fi/software/g3data.php.

\section{References}

[1] B. B. Aaron and J. M. Gosline. Elastin as a random-network elastomer: a mechanical and optical analysis of single elastin fibers. Biopolymers, 20:1247-1260, 1981.

[2] T. Belytschko, W. K. Liu, and B. Moran. Nonlinear Finite Elements for Continua and Structures. Wiley, Chichester, 2000.

[3] H. Braß. Quadraturverfahren, volume 3 of Studia Mathematica. Vandenhoeck \& Ruprecht, Göttingen, 1977.

[4] B. Budiansky and E. Kimmel. Elastic moduli of lungs. Journal of Applied Mechanics, 54:351-358, 1987.

[5] P. J. Dale, F. L. Matthews, and R. C. Schroter. Finite element analysis of lung alveolus. Journal of Biomechanics, 13:865-873, 1980.

[6] E. Denny and R. C. Schroter. The mechanical behavior of a mammalian lung alveolar duct model. Journal of Biomechanical Engineering, 117:254-261, 1995.

[7] E. Denny and R. C. Schroter. Relationships between alveolar size and fibre distribution in mammalian lung alveolar duct model. Journal of Biomechanical Engineering, 119:289-297, 1997.

[8] E. Denny and R. C. Schroter. Viscoelastic behavior of a lung alveolar duct model. Journal of Biomechanical Engineering, 122:143-151, 2000.

[9] A. Frankus and G. C. Lee. A theory for distortion studies of lung parenchyma based on alveolar membrane properties. Journal of Biomechanics, 7:101-107, 1974.

[10] A. D. Freed. Anisotropy in hypoelastic soft-tissue mechanics, I: theory. Journal of Mechanics of Materials and Structures, 3(5):911-928, 2008.

[11] A. D. Freed. Anisotropy in hypoelastic soft-tissue mechanics, II: simple extensional experiments. Journal of Mechanics of Materials and Structures, 4(6):1005-1025, 2009.

[12] A. D. Freed. Hypoelastic soft tissues, part I: theory. Acta Mechanica, 213:189-204, 2010.

[13] A. D. Freed. Soft solids: A primer to the theoretical mechanics of materials. In preparation, 2011.

[14] A. D. Freed, D. R. Einstein, and M. S. Sacks. Hypoelastic soft tissues, part II: in-plane biaxial experiments. Acta Mechanica, 213:205-222, 2010.

[15] A. D. Freed, D. R. Einstein, and I. Vesely. Invariant formulation for dispersed transverse isotropy in aortic heart valves: an efficient means for modeling fiber splay. Biomechanics and Modeling in Mechanobiology, 4:100-117, 2005.

[16] Y. C. Fung. Elasticity of soft tissues in simple elongation. American Journal of Physiology, 28:1532-1544, 1967.

[17] Y. C. Fung. Stress, deformation, and atelectasis of the lung. Circulation Research, 37:481-496, 1975. 
[18] Y. C. Fung. A model of the lung structure and its validation. Journal of Applied Physiology, 64:2132-2141, 1988.

[19] Y. C. Fung. Biomechanics: Motion, flow, stress, and growth. Springer-Verlag, New York, 1990.

[20] Y. C. Fung. Biomechanics: Circulation. Springer-Verlag, New York, second edition, 1996.

[21] C. J. Greenshields and H. G. Weller. A unified formulation for continuum mechanics applied to fluid-structure interaction in flexible tubes. International Journal for Numerical Methods in Engineering, 64:1575-1593, 2005.

[22] J. Hildebrandt. Pressure-volume data of cat lung interpreted by a plastoelastic, linear viscoelastic model. Journal of Applied Physiology, 28(3):365-372, 1970.

[23] R. Hill. On uniqueness and stability in the theory of finite elastic strain. Journal of the Mechanics and Physics of Solids, 5:229-241, 1957.

[24] R. Hill. A general theory of uniqueness and stability in elastic-plastic solids. Journal of the Mechanics and Physics of Solids, 6:236-249, 1958.

[25] G. A. Holzapfel. Nonlinear Solid Mechanics: A continuum approach for engineering. Wiley, Chichester, 2000.

[26] F. G. Hoppin, Jr. and J. Hildebrandt. Mechanical properties of the lung. In J. B. West, editor, Bioengineering Aspects of the Lung, volume 3 of Lung Biology in Health and Disease, pages 83-162. Marcel Dekker, New York, 1977.

[27] A. D. Karakaplan, M. P. Bieniek, and R. Skalak. A mathematical model of lung parenchyma. Journal of Biomechanical Engineering, 102:124-136, 1980.

[28] E. Kimmel and B. Budiansky. Surface tension and the dodecahedron model for lung elasticity. Journal of Biomechanical Engineering, 112:160-167, 1990.
[29] E. Kimmel, R. D. Kamm, and A. H. Shapiro. A cellular model of lung elasticity. Journal of Biomechanical Engineering, 109:126-131, 1987.

[30] S. J. Lai-Fook, T. A. Wilson, R. E. Hyatt, and J. R. Rodarte. Elastic constants of inflated lobes of dog lungs. Journal of Applied Physiology, 40(4):508-513, 1976.

[31] G. C. Lee and A. Frankus. Elasticity properties of lung parenchyma derived from experimental distortion data. Biophysical Journal, 15:481-493, 1975.

[32] A. S. Lodge. A network theory of flow birefringence and stress in concentrated polymer solutions. Transactions of the Faraday Society, 52:120-130, 1956.

[33] A. S. Lodge. Elastic Liquids: An introductory vector treatment of finite-strain polymer rheology. Academic Press, London, 1964.

[34] A. S. Lodge. Body Tensor Fields in Continuum Mechanics: With applications to polymer rheology. Academic Press, New York, 1974.

[35] M. Matsuda, Y. C. Fung, and S. S. Sobin. Collagen and elastin fibers in human pulmonary alveolar mouths and ducts. Journal of Applied Physiology, 63(3):1185-1194, 1987.

[36] J. C. Nadeau and M. Ferrari. Invariant tensorto-matrix mappings for evaluation of tensorial expressions. Journal of Elasticity, 52:43-61, 1998.

[37] W. Noll. On the continuity of the solid and fluid states. Journal of Rational Mechanics and Analysis, 4:3-81, 1955.

[38] J. G. Oldroyd. On the formulation of rheological equations of state. Proceedings of the Royal Society, London, A, 200:523-541, 1950.

[39] J. G. Oldroyd. Equations of state of continuous matter in general relativity. Proceedings of the Royal Society, London, A 316:1-28, 1970. 
[40] D. R. Otis Jr., E. P. Ingenito, R. D. Kamm, and M. Johnson. Dynamic surface tension of surfactant TA: experiments and theory. Journal of Applied Physiology, 77(6):2681-2688, 1994.

[41] A. D. Polyanin and V. F. Zaitsev. Handbook of Exact Solutions for Ordinary Differential Equations. Chapman \& Hall/CRC, Boca Raton, second edition, 2003.

[42] E. P. Radford, Jr. Recent studies of mechanical properties of mammalian lungs. In J. W. Remington, editor, Tissue Elasticity, pages 177-190, Baltimore, 1957. American Physiological Society, Waverly Press.

[43] S. Schürch, M.Lee, and P. Gehr. Pulmonary surfactant: surface properties and function of alveolar and airway surfactant. Pure and Applied Chemsitry, 64(11):1745-1750, 1992.

[44] J. C. Simo and T. J. R. Hughes. Computational Inelasticity, volume 7 of Interdisciplinary Applied Mathematics. Springer, New York, 1998.

[45] S. S. Sobin, Y. C. Fung, and H. M. Tremer. Collagen and elastin fibers in human pulmonary alveolar walls. Journal of Applied Physiology, 64(4):1659-1675, 1988.

[46] I. S. Sokolnikoff. Tensor Analysis: Theory and applications to geometry and mechanics of continua. Applied Mathematics Series. Wiley, New York, second edition, 1964.

[47] A. J. M. Spencer. Deformations in Fibre-reinforced Materials. Oxford Science Research Papers. Clarendon Press, Oxford, 1972.

[48] T. Sugihara, J. Hildebrandt, and C. J. Martin. Viscoelastic properties of alveolar wall. Journal of Applied Physiology, 33(1):93-98, 1972.

[49] S. P. Timoshenko and J. N. Goodier. Theory of Elasticity. Engineering Societies Monographs. McGraw-Hill, New York, third edition, 1970.

[50] L. R. G. Treloar. The Physics of Rubber Elasticity. Clarendon Press, Oxford, third edition, 1975.
[51] C. Truesdell. The mechanical foundations of elasticity and fluid dynamics. Journal of Rational Mechanics and Analysis, 2:593-616, 1953.

[52] C. Truesdell. Hypoelasticity. Journal of Rational Mechanics and Analysis, 4:83-133, 1955.

[53] C. Truesdell. The simplest rate theory of pure elasticity. Communications on Pure and Applied Mathematics, 8:123-132, 1955.

[54] C. Truesdell. Stages in the development of the concept of stress, volume Problems of Continuum Mechanics (Muskhelisvili Anniversary Volume), pages 556-564. Society of Industrial and Applied Mathematics, Philadelphia, 1961.

[55] D. W. Urry. Physicochemical properties of elastin and constituent peptides, volume 1 of Elastin and Elastases, pages 141-173. CRC Press, Boca Raton, 1989.

[56] D. L. Vawter. A finite element model for macroscopic deformation of the lung. Journal of Biomechanical Engineering, 102:1-7, 1980.

[57] E. R. Weibel and J. Gil. Structure-function relationships at the alveolar level. In J. B. West, editor, Bioengineering Aspects of the Lung, volume 3 of Lung Biology in Health and Disease, pages 1-81. Marcel Dekker, New York, 1977.

[58] J. B. West. Respiratory Physiology: The essentials. Lippincott Williams \& Wilkins, Philadelphia, eighth edition, 2007. 